Pacific

Journal of

Mathematics

\title{
MULTIPLICITY OF INVARIANT ALGEBRAIC CURVES IN POLYNOMIAL VECTOR FIELDS
}

Colin Christopher, Jaume Llibre And Jorge Vitório Pereira 


\title{
MULTIPLICITY OF INVARIANT ALGEBRAIC CURVES IN POLYNOMIAL VECTOR FIELDS
}

\author{
Colin Christopher, JAume Llibre And Jorge Vitório Pereira
}

The aim of this paper is to introduce a concrete notion of multiplicity for invariant algebraic curves in polynomial vector fields. In fact, we give several natural definitions and show that they are all equivalent to our main definition, under some "generic" assumptions.

In particular, we show that there is a natural equivalence between the algebraic viewpoint (multiplicities defined by extactic curves or exponential factors) and the geometric viewpoint (multiplicities defined by the number of algebraic curves which can appear under bifurcation or by the holonomy group of the curve). Furthermore, via the extactic, we can give an effective method for calculating the multiplicity of a given curve.

As applications of our results, we give a solution to the inverse problem of describing the module of vector fields with prescribed algebraic curves with their multiplicities; we also give a completed version of the Darboux theory of integration that takes the multiplicities of the curves into account.

In this paper, we have concentrated mainly on the multiplicity of a single irreducible and reduced curve. We hope, however, that the range of equivalent definitions given here already demonstrates that this notion of multiplicity is both natural and useful for applications.

\section{Introduction}

In 1878, Darboux published his seminal work on the integrability of polynomial differential equations in the plane. He showed how the integrability of a polynomial system could be obtained from an abundance of invariant algebraic curves. The idea was to construct an integrating factor for the system of the form

$$
\prod_{i=1}^{r} f_{i}^{l_{i}},
$$

MSC2000: 34C05, 34A34, 34C14.

Keywords: polynomial vector field, invariant algebraic curve, multiplicity, exponential factor,

Darboux integrability.

Pereira is partially supported by DGES grant number PB96-1153 and by CICYT grant number 1999SGR 00399. 
where the $f_{i}$ were polynomials defining the invariant algebraic curves of the system. Although this might be seen as a rather special case, it was shown in [Prelle and Singer 1983] that such integrating factors account for all systems with elementary first integrals.

However, in order to find a natural completion of Darboux's theory, it was found necessary to consider the possibility that certain of the algebraic curves coalesce (see [Christopher 1994] for example). When this happens, these "multiple" curves give rise to exponential factors in the Darboux integrating factor of the form

$$
\prod_{i=1}^{r} f_{i}^{l_{i}} \prod_{j=1}^{s} \exp \left(g_{j} / h_{j}\right),
$$

where the functions $\exp \left(g_{i} / h_{i}\right)$ satisfy an equation similar to the polynomials $f_{i}$ that define the invariant algebraic curves $f_{i}=0$. We call such a function Darboux, and the terms $\exp \left(g_{i} / h_{i}\right)$ exponential factors. It has been shown by Singer [1992] (with the additional comments from [Christopher 1999], for example) that polynomial vector fields with a Darboux integrating factors are exactly those with Liouvillian first integrals.

Although the heuristic equivalence between the coalescence of algebraic solutions and the rise of exponential factors has now been used by several authors, there has been no general theory given beyond ad hoc comments like those of [Christopher 1994]. In particular, the case where several curves coalesce and form exponential factors has been mostly ignored.

The difficulty is that it is not obvious, given a polynomial vector field, to see whether any given invariant algebraic curve is 'multiple' or not, nor how to assign it a multiplicity. Clearly the usefulness of this goes beyond the Darboux theory to any problem dealing with families of polynomial systems with several algebraic curves. Such families arise very naturally: for example when looking at families of vector fields with centers or other integrable critical points, where many of the irreducible components of these families have generically a Darboux first integral or integrating factor, and it is interesting to know what happens for the nongeneric vector fields in these components.

Our aim in this paper is to give a concrete definition of multiplicity which is effectively computable and show that, given some 'generic' assumptions, there is an equivalence between this definition and several other natural definitions which we will indicate below.

Throughout the paper, therefore, we have concentrated only on the "generic" case. By this, we mean that we only consider the multiplicity of an irreducible and reduced curve. That is, a curve given by an irreducible polynomial. For more general curves, there seem to be several competing definitions of multiplicity, and we plan to treat these cases in a future paper. It is our hope, however, that although 
in a somewhat restricted setting, the results given here are sufficient to show that this definition of multiplicity is indeed a very natural and useful one.

We shall sketch, briefly, the contents of the paper, and the notions of multiplicity considered in the text.

In Section 2, we give a short overview of the basic results and methods using invariant algebraic curves for polynomial vector fields, with the aim of giving some sort of orientation to the rest of the paper. Most of the results given in this section are entirely standard and well-known.

In Section 3 we give our main definition of multiplicity, which expresses the fact that the existence of a multiple curve involves not just the curve but also some infinitesimal information about it. We call this infinitesimal multiplicity when we need to distinguish it from the other definitions below, but otherwise we shall drop the adjective.

Let $\varepsilon$ be an algebraic quantity with $\varepsilon^{k}=0$ (or, equivalently, we consider $\varepsilon$ as lying in the ring $\left.\mathbb{C}(\varepsilon) /\left(\varepsilon^{k}\right)\right)$; we say a curve $f=0$ has multiplicity $k$ if there exist polynomials $f_{0}=f, f_{1}, \ldots, f_{k-1}$, of degree no more than the degree of $f$, such that $F=f_{0}+\varepsilon f_{1}+\cdots+\varepsilon^{k-1} f_{k-1}$ satisfies

$$
X(F)=F L_{F}
$$

in $\mathbb{C}[x, y, \varepsilon] /\left(\varepsilon^{k}\right)$, where $X$ is the vector field, and $L_{F}$ lies in $\mathbb{C}[x, y, \varepsilon] /\left(\varepsilon^{k}\right)$ also. When $k=1$, this is just the defining equation of a single invariant algebraic curve. If $f_{1}$ is not a multiple of $f_{0}$, then we say that $F$ is nondegenerate. The infinitesimal multiplicity is then the maximum of the $k$ 's for which such a nondegenerate $F$ exists.

We then go on to consider briefly some local consequences of this definition, and to derive bounds on the multiplicity of a curve based on the formal normal forms of the critical points on the curve.

Section 4 defines multiplicity in terms of the number of exponential factors associated with the curve $f=0$. This is the feature which has been found so useful in applications. A curve $f=0$ is said to be of integrable multiplicity $k$ if it gives rise to $k-1$ exponential factors in the form $\exp \left(g_{j} / f^{j}\right), j=1, \ldots, k-1$, with the degree of $g_{j}$ at most $j$ times the degree of $f$, and such that $f$ is not a factor of $g_{j}$. These exponential factors can be used in the construction of Darboux integrating factors, first integrals, or linearizing changes of coordinates. We show that, under the assumption that $f=0$ be irreducible and reduced, this definition coincides with the infinitesimal multiplicity above.

Section 5 gives another purely algebraic definition of multiplicity. In [Pereira 2001] were introduced the extactic curves for polynomials vector fields. Essentially, these are curves of higher-order inflection points for a given vector field. A nice feature of these curves is that they can be calculated directly from the 
determinant of a matrix. A curve $f=0$ is said to have algebraic multiplicity $k$ if $f^{k}$ is a factor in this determinant; and hence the algebraic multiplicity is effectively computable. Again, we show that this definition is equivalent to infinitesimal multiplicity under the assumption that $f=0$ be irreducible and reduced.

Section 6 defines multiplicity in geometric terms: we say that a curve $f=0$ has geometric multiplicity $k$ if there are arbitrarily small perturbations of the vector field which give rise to $k$ invariant algebraic curves, bifurcating from $f=0$. To make this concept more supple in applications, we allow perturbations in the class of rational vector fields as well as polynomial ones. However, we give a sufficient condition for the perturbation to be realized polynomially. It is a long section, as we digress slightly to solve the inverse problem of describing the space of vector fields with prescribed multiple invariant algebraic curves. This is a result of independent interest, however, we use it here to show that the geometric multiplicity is equivalent to the infinitesimal multiplicity, under the previous assumption on $f$.

Our final definition, in Section 7, is more geometric still. Given an algebraic curve, we can define the holonomy group of the foliation induced by the vector field in a neighborhood of the curve. A curve is said to be of holonomic multiplicity $k$ if all the elements of its holonomy group are tangent to the identity, up to order $k$. Its clear that this is a necessary condition for having the possibility of bifurcating multiple algebraic curves, but without some underlying assumptions this notion of multiplicity is much weaker than geometric multiplicity. However, if we assume that the curve $f=0$ is nonsingular, as a projective curve, and make some assumptions on the critical points which lie on $f=0$, we can show that holonomic multiplicity is equivalent to the other multiplicities.

In the final Section 9, we give a small application, by completing Darboux's theorem on integrability to include the case of multiple curves, and add some final remarks.

Main Theorem. If an invariant algebraic curve $f=0$ is irreducible and reduced (that is, the polynomial $f$ does not factorize at all), then the following multiplicities are equivalent:

- the infinitesimal multiplicity of $f=0$;

- the integrable multiplicity of $f=0$;

- the algebraic multiplicity of $f=0$;

- the geometric multiplicity of $f=0$.

If , in addition, the curve $f=0$ is nonsingular (as a projective curve) and all the critical points which lie on $f=0$ are regular with respect to $f=0$, including the ones at infinity, then the above multiplicities are also equivalent to

- the holonomic multiplicity of $f=0$. 
Here, a critical point on $f=0$ is said to be regular with respect to $f=0$ if it is either hyperbolic with a positive ratio of eigenvalues, or it is a saddle node and the eigenvalue associated to the direction tangent to $f=0$ is the nonzero one.

A number of authors have considered how to define the multiplicity of a curve. Żołądek [1992] gives a definition in terms of the local multiplicities at each critical point. Unfortunately, the definition is not sufficiently strong to guarantee that this number of curves can actually be produced by bifurcation. This definition would seem to be closest to holonomic multiplicity, but only considered locally.

Schlomiuk [1997] defines a notion of geometric multiplicity of an invariant algebraic curve with respect to a particular family of polynomial vector fields. However, no effective means is given for calculating this multiplicity apart from inspecting the family itself. This definition is closest in spirit to the definition of geometric multiplicity given here.

A simplified version of the chain of equations which underlie the definition of infinitesimal multiplicity was considered in Gröbner and Knapp [1967]. Of course, the concept of curves and other objects which include infinitesimal information about themselves is an essential component of modern algebraic geometry. However, although interesting, we do not pursue this relationship here.

\section{Background and preliminaries}

Before embarking on our main journey, we want to summarize briefly the way that invariant algebraic curves have been defined up to now, and the use that has been made of them in the Darboux theory on integration, etc. In doing so, we hope that the subsequent sections will be put in a clearer context.

\section{A. Vector fields.}

Definition 2.1. We say that $X$ is a (polynomial) vector field of (affine) degree $d$ on $\mathbb{C}^{2}$ if it can be written in the form

$$
X=a(x, y) \frac{\partial}{\partial x}+b(x, y) \frac{\partial}{\partial y},
$$

where $a(x, y)$ and $b(x, y)$ are polynomials in $\mathbb{C}[x, y]$ such that the maximum degree of $a$ and $b$ is $d$.

We shall always assume that $X$ is nondegenerate or reduced; that is, there is no nontrivial polynomial dividing $a$ and $b$.

A different notion of degree of a vector field is this: $X$ has (projective) degree $d$ if the number of tangencies of $X$ with a generic straight line is $d$. This second definition has the advantage that the family of systems of degree $d$ are invariant under projective transformations (after a time-scaling). In general, the two definitions do not coincide. However, a vector field of degree $d$ in this second sense can 
always be put into the form

$$
X=(a(x, y)+x h(x, y)) \frac{\partial}{\partial x}+(b(x, y)+y h(x, y)) \frac{\partial}{\partial y},
$$

where $a$ and $b$ have degree $d$ as before, and $h$ is a homogeneous polynomial of degree $d$.

The system (2-2) reduces to (2-1) if and only if $h=0$; this corresponds, geometrically, to the line at infinity of (2-2) being invariant. In fact, a vector field of the form (2-2) with an invariant line can be brought to the form (2-1) by a projective transformation (and a time-scaling) which takes the invariant line to the line at infinity. Conversely, if we want to understand the role of the line at infinity of (2-1), we can perform a projective transformation to bring the line at infinity to an invariant line in the finite plane. In this case, the vector field (2-1) is brought to the form (2-2) (after a time-scaling). For more details about this, see [Lins Neto and Scárdua 1997].

Unless otherwise stated, we shall consider the affine case.

\section{B. Invariant algebraic curves.}

Definition 2.2. Let $f \in \mathbb{C}[x, y]$. If the algebraic curve $f=0$ is invariant by a vector field $X$ of degree $d$, then $X(f) / f$ is a polynomial of degree at most $d-1$. In this case we say that $f=0$ is an invariant algebraic curve of $X$ and $L_{f}=X(f) / f$ is its cofactor.

An algebraic curve $f=0$ is said to be irreducible if it has only one component, and reduced if all components appear with multiplicity one (that is, the polynomial $f$ is square-free). Clearly, a curve $f=0$ is irreducible and reduced if and only if $f$ is irreducible as a polynomial.

Note that, if the vector field $X$ has several invariant algebraic curves of different degrees, the cofactors will all lie in $\mathbb{C}_{d-1}[x, y]$, the vector space of polynomials of degree at most $d-1$. This allows us to reduce the problem of Darboux integrability to one of linear algebra. The next two propositions can be found in [Christopher and Llibre 2000].

Proposition 2.3. Let $f \in \mathbb{C}[x, y]$ and $f=f_{1}^{n_{1}} \ldots f_{r}^{n_{r}}$ be its factorization in irreducible factors. For a vector field $X, f=0$ is an invariant algebraic curve with cofactor $L_{f}$ if and only if $f_{i}=0$ is an invariant algebraic curve for each $i=1, \ldots, r$ with cofactor $L_{f_{i}}$. Moreover, $L_{f}=n_{1} L_{f_{1}}+\cdots+n_{r} L_{f_{r}}$.

Definition 2.4. Given $f, g \in \mathbb{C}[x, y]$, we say that $e=\exp (g / f)$ is an exponential factor of the vector field $X$ of degree $d$ if $X(e) / e$ is a polynomial of degree at most $d-1$. This polynomial is called the cofactor of the exponential factor $e$, which we denote by $L_{e}$. The quotient $g / f$ is an exponential coefficient of $X$. 
Proposition 2.5. If $e=\exp (g / f)$ is an exponential factor for the vector field $X$, then $f$ is an invariant algebraic curve and $g$ satisfies the equation

$$
X(g)=g L_{f}+f L_{e},
$$

where $L_{f}$ is the cofactor of $f$.

Example 2.6. Consider the vector field

$$
X=x \frac{\partial}{\partial x}+((1+\ell) y+x) \frac{\partial}{\partial y}
$$

with invariant algebraic curves $x=0$ and $x+\ell y=0$. As $\ell$ tends to zero, these two curves coalesce. However, we can recover an exponential factor by taking the limit of the Darboux function $((x+\ell y) / x)^{1 / \ell}$, which tends to $\exp (y / x)$.

In general, we would hope that an exponential factor $\exp (f / g)$ corresponds to the coalescence, as $\ell$ tends to zero, of two invariant algebraic curves $f=0$ and $f+\ell g=0$. That is, we consider $\exp (f / g)$ as

$$
\exp (f / g)=\lim _{\ell \rightarrow 0}((f+\ell g) / f)^{1 / \ell} .
$$

However, to show that this is always the case is quite difficult. The following result is quoted in [Christopher and Llibre 1999]:

Proposition 2.7. If a vector field $X$ has an exponential factor $\exp (g / f)$, where $g=0$ and $f=0$ are nonsingular and have normal crossings with themselves and the line at infinity, then the vector field is of the form

$$
\left(a_{0} f^{2}-a_{1} f f_{y}-a_{2}\left(g_{y} f-g f_{y}\right)\right) \frac{\partial}{\partial x}+\left(a_{3} f^{2}+a_{1} f f_{x}+a_{2}\left(g_{x} f-g f_{x}\right)\right) \frac{\partial}{\partial y} .
$$

In particular, the exponential can be seen to be the limit of the invariant curves $f$ and $f+\epsilon g$ as $\epsilon$ tends to zero in the family of vector fields $X_{\epsilon}=p_{\epsilon} \partial / \partial x+q_{\epsilon} \partial / \partial y$, where

$$
\begin{aligned}
& p_{\epsilon}=a_{0} f(f+\epsilon g)-\left(a_{1}+\epsilon^{-1} a_{2}\right) f(f+\epsilon g)_{y}+\epsilon^{-1} a_{2} f_{y}(f+\epsilon g), \\
& q_{\epsilon}=a_{3} f(f+\epsilon g)+\left(a_{1}+\epsilon^{-1} a_{2}\right) f(f+\epsilon g)_{x}-\epsilon^{-1} a_{2} f_{x}(f+\epsilon g),
\end{aligned}
$$

which tends to (2-4) as $\epsilon$ tends to zero.

The results of this paper can be seen as a direct generalization of the examples above to multiple coalescing curves of arbitrary degree. 
2C. The line at infinity. Though we shall concentrate mainly on the affine case, it is often helpful to consider the behavior on the line at infinity.

Consider the vector field (2-1) and write the terms of degree $d$ in $a$ and $b$ as $a_{d}$ and $b_{d}$. If $r=x b_{d}-y a_{d}$ vanishes, then the system is equivalent to a vector field (2-2) of projective degree $d-1$. If $r \neq 0$ however, then the line at infinity is invariant, and the zeros of $r$ correspond to the critical points of (2-1) on the line at infinity.

The next lemma is well known (see for example [Christopher 1994]) and can be very useful computationally (see [Man and MacCallum 1997] for an interesting application).

Lemma 2.8. Let $X$ be a vector field of the form (2-1) with invariant algebraic curve $f=0$ of degree $n$. Each factor of the highest-order terms $f_{n}$ of $f$ divides $r=x b_{d}-y a_{d}$.

If, however, we consider a vector field in the form (2-2), then the line at infinity is no longer invariant. Nevertheless, in this case we have the next proposition.

Proposition 2.9. Suppose the vector field $X$ is in the form (2-2), of projective degree $d$, with $h \neq 0$.

(a) If $L_{f}$ is the cofactor of an invariant algebraic curve $f=0$ of $X$, then the degree-d terms of $L_{f}$ are given by $\operatorname{deg}(f) h$.

(b) If $L_{e}$ is the cofactor of an exponential factor $e=\exp (g / f)$ of $X$, then the degree-d terms of $L_{e}$ vanish.

Proof. Let $f$ be of degree $n$, and denote the degree- $n$ terms of $f$ by $f_{n}$. Using the notation of (2-2) and Euler's formula, the equation $X(f)=L_{f} f$ gives

$$
a \frac{\partial f}{\partial x}+b \frac{\partial f}{\partial y}+h\left(x \frac{\partial}{\partial x}+y \frac{\partial}{\partial y}\right)\left(f-f_{k}\right)+k h f_{k}=L_{f} f .
$$

The result of part (a) follows directly from considering the degree- $(n+d)$ terms of this equation.

For part (b), we let $\ell$ be the degree of $g$, and $g_{\ell}$ the terms of degree $\ell$. It follows, from the defining equations for an exponential factor, that

$$
(a+h x)\left(f \frac{\partial g}{\partial x}-g \frac{\partial f}{\partial x}\right)+(b+h y)\left(f \frac{\partial g}{\partial y}-g \frac{\partial f}{\partial y}\right)=L_{e} f^{2} .
$$

The terms of highest degree $n+\ell+d$ on the left-hand side of (2-5) can be written as

$$
h\left(x\left(f_{n} \frac{\partial g_{\ell}}{\partial x}-g_{\ell} \frac{\partial f_{n}}{\partial x}\right)+y\left(f_{n} \frac{\partial g_{\ell}}{\partial y}-g_{\ell} \frac{\partial f_{n}}{\partial y}\right)\right)=(\ell-k) h f_{k} g_{\ell} .
$$


It is clear that if the degree- $d$ terms of $L_{e}$ do not vanish, then the degree of the righthand side of (2-5) is $2 n+d$, thus we must have $\ell=k$, which gives a contradiction.

In the projective case, therefore, the exponential exponents are always homogeneous of degree 0 . In the affine case of (2-1) this restriction no longer holds, but, if the degree of the numerator is greater than the degree of the denominator, then, after a projective change of coordinates, the exponential factor will be of the form $\exp \left(g /\left(f m^{k}\right)\right)$ for some $k>0$, where $m$ represents the transform of the line at infinity. Thus, this case corresponds projectively to the case where the exponential factor is based on the line at infinity or some reducible combination of the line at infinity with another curve. In this paper we shall only consider exponential factors based on irreducible algebraic curves in the finite plane, thus the degrees of the numerators of the exponential coefficients must be less than their denominators. The line at infinity can be considered in this manner if a projective transformation is performed first to bring it into the finite plane.

2D. Integrating factors and first integrals. One of the main applications of invariant algebraic curves is in constructing first integrals and integrating factors of Darboux type; that is, functions which are expressible as products of invariant algebraic curves and exponential factors. We recall a few definitions.

Definition 2.10. Let $P / Q$ be a rational function in $x$ and $y$, with $P$ and $Q$ coprime; its degree is the maximum of the degrees of $P$ and $Q$.

Definition 2.11. A (multivalued) function is said to be Darboux if it is of the form

$$
\prod_{i=1}^{r} f_{i}^{l_{i}} \prod_{j=1}^{s} \exp \left(g_{j} / h_{j}\right),
$$

where the $f_{i}, g_{j}$ and $h_{j}$ are polynomials, and the $l_{i}$ are complex numbers. The set of such functions is precisely the set of exponentials of integrals of closed rational 1 -forms in $x$ and $y$ ([Christopher 1999]).

Definition 2.12. Let $U$ be an open subset of $\mathbb{C}^{2}$. We say that a nonconstant (multivalued) function $H: U \rightarrow \mathbb{C}$ is a first integral of a vector field $X$ on $U$ if and only if

$$
\left.X\right|_{U}(H)=0 .
$$

When $H$ is the restriction of a rational (respectively, Darboux) function to $U$ then we say that $H$ is a rational (respectively, Darboux) first integral.

Definition 2.13. We say that a nonconstant (multivalued) function $R: U \rightarrow \mathbb{C}$ is an integrating factor of a vector field $X$ on $U$ if and only if

$$
X(R)=-\operatorname{div} X \cdot R
$$


on $U$, where div denotes the divergence of the vector field.

If we know an integrating factor, we can compute by quadrature a first integral of the system, up to a constant. Reciprocally, if $H$ is a first integral of the vector field (2-1), then there is a unique integrating factor $R$ satisfying

$$
R a=\frac{\partial H}{\partial y} \quad \text { and } \quad R b=-\frac{\partial H}{\partial x} .
$$

Such an $R$ is called the integrating factor associated to $H$.

A theorem of Singer [1992] shows that, if $H$ is a Liouvillian function, then the integrating factor is Darboux. In an earlier work, Prelle and Singer [1983] show that if $H$ is an elementary function, then the integrating factor is the $N$-th root of a rational function.

The idea behind the Darboux method is to use the invariant algebraic curves of the system to find an integrating factor of the form (2-6). This, in turn, from Proposition 2.3, is purely a matter of linear algebra, as all the cofactors lie in $\mathbb{C}_{d-1}[x, y]$. A simple introduction to these things can be found in [Christopher and Llibre 1999].

For example, we can find a Darboux first integral (2-6) if we can find constants $l_{i}$ and $m_{i}$ such that

$$
\sum_{i=1}^{r} l_{i} L_{f_{i}}+\sum_{j=1}^{s} m_{j} L_{e_{j}}=0
$$

where the $L_{f_{i}}$ and $L_{e_{j}}$ represent the cofactors of $f_{i}$ and $\exp \left(g_{j} / h_{j}\right)$, respectively. In particular, this will always happen if there are more than $d(d+1) / 2$ such curves or exponential factors.

In practice, the number of curves needed may be much less than this, and the method can be refined by considering critical points where the curves $f_{i}$ do not vanish.

Proposition 2.14. Let $p$ be a critical point of the vector field $X$. If $f$ is an invariant algebraic curve of $X$ which does not vanish at $p$, then its cofactor $L_{f}$ must vanish at $p$. Furthermore, if $e=\exp (g / f)$ is an exponential factor of $X$, then $L_{e}$ must vanish at $p$ too.

Proof. This follows directly from the equations $X(f)=L_{f} f$ and $X(g)=L_{f} g+$ $L_{e} f$.

We shall generalize these results to multiple curves in Section 3B.

2E. Formal normal forms for elementary critical points. If $p$ is a noncritical point of an analytic vector field, then it is well known that there is an analytic change of coordinates in a neighborhood of $p$ which brings the vector field into the form $X=\partial / \partial x$. If $p$ is a critical point, however, the analytic classification is 
much harder. In this paper we shall only want to consider the formal classification of elementary singularities. That is, critical points with at least one nonzero eigenvalue.

The following formal normal forms for elementary singularities are well known [Poincaré 1891; 1897].

Theorem 2.15. Let $X$ be a vector field with an isolated critical point at the origin. There is a formal analytic change of coordinates at the origin which brings $X$ to one of the following forms, after multiplication by a unit in $\mathbb{C} \llbracket x, y \rrbracket$ :

(a) A linearizable critical point $(\lambda \neq 0)$,

$$
X=x \frac{\partial}{\partial x}+\lambda y \frac{\partial}{\partial y}
$$

(b) A resonant node $\left(k \in \mathbb{Z}_{+}\right)$,

$$
X=x \frac{\partial}{\partial x}+\left(k y+x^{k}\right) \frac{\partial}{\partial y}
$$

(c) A resonant saddle $\left(p, q, k \in \mathbb{Z}_{+}, a \in \mathbb{C}\right)$,

$$
X=x\left(1+a u^{k}\right) \frac{\partial}{\partial x}-\frac{p}{q} y\left(1+(a-1) u^{k}\right) \frac{\partial}{\partial y}, \quad u=x^{p} y^{q}
$$

(d) A saddle-node $(k \in \mathbb{Z}, a \in \mathbb{C})$,

$$
X=x\left(1+a y^{k}\right) \frac{\partial}{\partial x}+y^{k+1} \frac{\partial}{\partial y}
$$

In each of these cases, the only irreducible invariant algebroid (formal analytic) branches through the origin are $x=0$ (all cases), $y=0$ (all cases except the resonant node), and $x^{p}-c y^{q}$ for some constant $c$ (for the linearizable critical point with $\left.\lambda=p / q \in \mathbb{Q}_{+}\right)$.

The reason for considering only elementary singularities is that any other singularity of the vector field can be transformed into a collection of elementary singularities after a finite number of blow-ups (see [van den Essen 1979]). In what follows we do not need to work over the more general algebraic surfaces that arise after blowing up a critical point: we only need to know the behavior in a collection of affine charts which describe it, and these can be obtained very simply by a succession of affine transformations and transformations of the form $(u, v)=(x, y / x)$.

\section{Infinitesimal multiplicity}

We give our main definition of multiplicity for an invariant algebraic curve $f=0$ of degree $n$. We shall assume throughout that the algebraic curve $f=0$ is reduced and 
irreducible (that is, $f$ is an irreducible polynomial). We shall also assume that $X$ is a vector field of (affine) degree $d$ which acts nontrivially on $f=0$, by which we mean that the restriction of $X$ to $f=0$ does not vanish identically (equivalently, $f$ is not a factor of both $a$ and $b$ in (2-1)). This implies in particular that, if $X(g)=0$ on $f=0$ for some function $g$, then $g$ must be constant on $f=0$.

In Section 3B we deduce some elementary bounds on the infinitesimal multiplicity from the type of singularities through which the curve passes. These bounds are similar to the ones given in [Żoładek 1992].

\section{A. Definition of infinitesimal multiplicity.}

Definition 3.1. Let $f=0$ be an invariant algebraic curve of degree $n$ of a polynomial vector field $X$ of degree $d$. We say that

$$
F=f_{0}+f_{1} \varepsilon+\cdots+f_{k-1} \varepsilon^{k-1} \in \mathbb{C}[x, y, \varepsilon] /\left(\varepsilon^{k}\right)
$$

defines a generalized invariant algebraic curve of order $k$ based on $f=0$ if $f_{0}=$ $f, \ldots, f_{k-1}$ are polynomials in $\mathbb{C}[x, y]$ of degree at most $n$, and $F$ satisfies the equation

$$
X(F)=F L_{F},
$$

for some polynomial

$$
L_{F}=L_{0}+L_{1} \varepsilon+\cdots+L_{k-1} \varepsilon^{k-1} \in \mathbb{C}[x, y, \varepsilon] /\left(\varepsilon^{k}\right),
$$

which must necessarily be of degree at most $d-1$ in $x$ and $y$. We call $L_{F}$ the cofactor of $F$. Equivalently, (3-2) can be written as

$$
X\left(f_{i}\right)=f_{i} L_{0}+f_{i-1} L_{1}+\cdots+f_{0} L_{i}, \quad i=0, \ldots, k-1 .
$$

Note that, at the moment, we do not make any assumption that the $f_{i}$ are nonzero for $i>0$. If we want to make the role of $\varepsilon$ explicit, we shall use the notation $F(\varepsilon)$. The choice of notation in Definition 3.1 will be assumed in the rest of the paper, unless otherwise stated. We shall also use the notation $O\left(\varepsilon^{2}\right)$, etc., in the obvious sense.

Example 3.2. A generalized invariant algebraic curve of order 2 is given by two polynomials $f_{0}$ and $f_{1}$ satisfying the equations

$$
X\left(f_{0}\right)=f_{0} L_{0} \quad \text { and } \quad X\left(f_{1}\right)=f_{1} L_{0}+f_{0} L_{1},
$$

for some polynomials $L_{0}$ and $L_{1}$ in $x$ and $y$. These define an invariant algebraic curve $f_{0}$ and an exponential factor $\exp \left(f_{1} / f_{0}\right)$ of $X$ with cofactors $L_{0}$ and $L_{1}$, respectively. 
Given a generalized invariant algebraic curve $F$ of order $k$, and an element $\ell$ of $\mathbb{C}[\varepsilon] /\left(\varepsilon^{k}\right)$, it follows directly from (3-2) that $\ell F$ is also a generalized invariant algebraic curve of order $k$. Also, if $h$ is a polynomial over $\mathbb{C}$ with $h(\varepsilon)=\varepsilon+O\left(\varepsilon^{2}\right)$, then it follows that $h(\varepsilon)^{k}=0$, and (3-2) implies that $F^{\prime}(\varepsilon)=F(h(\varepsilon))$ also defines a generalized invariant algebraic curve of order $k$.

We would like to measure the multiplicity of an invariant algebraic curve $f=0$ by considering the maximum order of all the generalized invariant algebraic curves which are based on $f$. However, given a generalized curve $F$ of order $k$, we can form a new generalized curve $F^{\prime}$ of order $k q$ by taking $F^{\prime}(\delta)=F\left(\delta^{q}\right) \in$ $\mathbb{C}[x, y, \delta] /\left(\delta^{k q}\right)$. To exclude these cases, we need to make another definition.

Definition 3.3. A generalized algebraic curve $F$ based on $f$ is called nondegenerate if the polynomial $f_{1}$ from Definition 3.1 is not a multiple of $f$. Otherwise, we say that the curve is degenerate.

In fact, this definition turns out to exclude only the cases mentioned above, as the next proposition shows. Note that the degree bounds on the $f_{i}$ are needed in the proof.

Proposition 3.4. Let $F$ be a generalized invariant algebraic curve of order $k$ based on $f$. If $F$ is not a multiple of $f$, then there exist elements

$$
\ell=1+O(\varepsilon) \quad \text { and } \quad h=\varepsilon+O\left(\varepsilon^{2}\right)
$$

of $\mathbb{C}[\varepsilon]$ and a nondegenerate generalized invariant algebraic curve $F^{\prime}$ such that

$$
\ell(\varepsilon) F(h(\varepsilon))=F^{\prime}\left(\varepsilon^{q}\right)
$$

over $\mathbb{C}[x, y, \varepsilon] /\left(\varepsilon^{k}\right)$ for some integer $q>0$.

Proof. By multiplying $F$ by a suitable element of $\mathbb{C}[\varepsilon] /\left(\varepsilon^{k}\right)$, we can first remove all the $f_{i}$ which are multiples of $f_{0}$. If $F$ is not a multiple of $f_{0}=f$, there is some $q>0$ such that $f_{q}$ is the first nonzero $f_{i}$ after $f_{0}$ in $F(\varepsilon)$. If $q=1$, then we are done, so we assume that $q>1$. Now, if all the nonzero $f_{i}$ have $i$ a multiple of $q$, then we are also finished, so we shall suppose that there exists some $f_{r}$ such that $r$ is the first nonmultiple of $q$ with $f_{r}$ nonzero, and proceed by induction on $r$.

From (3-4) we find that

$$
X\left(f_{q}\right)=L_{0} f_{q}+L_{q} f_{0} \quad \text { and } \quad X\left(f_{r}\right)=L_{0} f_{r}+L_{r} f_{0},
$$

from which we deduce that $X\left(f_{q} / f_{r}\right)=0$ on $f_{0}=0$. Since $f_{0}=0$ is an irreducible curve on which $X$ is a nontrivial vector field, we see that $f_{q}=a f_{r}$ for some constant $a$ on $f_{0}=0$. Comparing degrees, we find that $f_{q}=a f_{r}+b f_{0}$ for some constant $b$. Taking $\ell=1-b \varepsilon^{r}$ and $h=\varepsilon-a \varepsilon^{r-q+1}$, we can replace $F$ by $\ell(\varepsilon) F(h(\varepsilon))$, which coincides with $F$ up to the term in $\varepsilon^{r-1}$ and has no term in $f_{r}$. We repeat 
the above procedure until all remaining terms are of the form $f_{i q}$. The resulting transformation of $F$ is clearly of the prescribed form.

Definition 3.5. Let $f=0$ be an invariant algebraic curve of degree $n$ in a polynomial vector field $X$ of degree $d$. We say that $f=0$ is of infinitesimal multiplicity $m$ with respect to $X$ if $m$ is the maximal order of all nondegenerate generalized invariant algebraic curves of $X$ based on $f$. If no such maximum exists, then the infinitesimal multiplicity is said to be infinite.

It will follow from the results in Section 5 that, if the vector field has an invariant algebraic curve $f=0$ of degree $n$ which has infinite infinitesimal multiplicity, then the vector field has a rational first integral of degree $n$. In fact, we can choose it to be of the form $g / f$, for some polynomial $g$ of degree at most $n$. Conversely, a rational first integral $g / f$ of degree $n$ will give a curve $f=0$ of infinite infinitesimal multiplicity by taking the generalized invariant algebraic curve $F=f+\varepsilon g$ with cofactor $L_{F}=L_{f}(1+\varepsilon)$ which satisfies (3-2) up to arbitrary order of $\varepsilon$.

Question 3.6. It not clear to what extent Proposition 2.3 can be generalized to multiple curves. For example, if $F=F_{1} F_{2}$ is a generalized invariant algebraic curve of order $k$ (with factorization over $\mathbb{C}[x, y, \varepsilon] /\left(\varepsilon^{k}\right)$ ), can we conclude that each of the $F_{i}$ have order $k$ too?

We shall, however, give the following weak version of Proposition 2.3, which will be useful in the next section.

Proposition 3.7. Let $F$ be a generalized invariant algebraic curve of order $k$ and suppose that $F$ factorizes into $F_{1} F_{2}$ over $\mathbb{C}[x, y, \varepsilon] /\left(\varepsilon^{k}\right)$. If $F_{1}$ is an invariant algebraic curve of order at least $k$, then so is $F_{2}$.

Proof. We have

$$
F L_{F}=X(F)=F_{1} L_{F_{1}} F_{2}+F_{1} X\left(F_{2}\right),
$$

whence

$$
F_{1}\left(X\left(F_{2}\right)-\left(L_{F}-L_{F_{1}}\right) F_{2}\right)=0,
$$

and the result follows directly.

Note that we do not impose that $F_{1}$ be nondegenerate.

We can consider the generalized curve $F$ as an infinitesimal family of curves defined over the base space $T=\operatorname{Spec}\left(\mathbb{C}[\varepsilon] /\left(\varepsilon^{k}\right)\right)$ with $f$ the fibre over $0 . \quad F$ is therefore an infinitesimal deformation of $f$ over $T$. We do not pursue this algebraic geometric language here, however.

3B. Local bounds on multiplicity. It is an interesting feature of the algebraic theory of vector fields that most of the action seems to happen at the critical points. We give some simple bounds on the infinitesimal multiplicity of a curve, depending only on the formal classification of the critical points it passes through. A similar 
criterion was given by Żołądek in his definition of multiplicity [Żołądek 1992]. We start, however, with a couple of simple propositions which will be useful later.

We shall say that a generalized invariant algebraic curve $F=f_{0}+O(\varepsilon)$ passes through a point $p$ if $f_{0}(p)=0$. In the proofs that follow we will mainly want to work locally around a point $p$. To do so, we can assume without loss of generality that $p$ is at the origin and work over $\mathbb{C} \llbracket x, y \rrbracket[\varepsilon] /\left(\varepsilon^{k}\right)$. The analogous definitions of (generalized) invariant algebroid curve of order $k$ and the corresponding translation of (3-2) and the preceding propositions to this context are left to the reader.

Clearly, a generalized algebraic curve of order $k$ passing through the point $p$ gives rise to a generalized algebroid curve of order $k$ at $p$. However, in general we need to be careful that, although we are assuming that the curve $f_{0}=0$ is irreducible and reduced over $\mathbb{C}[x, y]$, it may split into a number of algebroid branches over $\mathbb{C} \llbracket x, y \rrbracket$, though none of these branches can be multiple.

Proposition 3.8. Let $F=f_{0}+\varepsilon f_{1}+O\left(\varepsilon^{2}\right)$ be a nondegenerate generalized invariant algebraic curve of a vector field $X$. If $p$ is a noncritical point of $X$ for which $f_{0}(p)=0$, then $p$ is a nonsingular point of $f_{0}=0$ and $f_{1}$ does not vanish on $p$.

Proof. Without loss of generality, we can assume that $p$ lies at the origin; we perform a local (formal) change of coordinates in $\mathbb{C} \llbracket x, y \rrbracket$ which brings $X$ to the form $\partial / \partial x$. If $f_{0}(p)=0$, then $y$ must be a factor of $f_{0}=0$. In this case, we let $f_{0}^{\prime}=f_{0} / y$ and consider the invariant algebroid curve $f_{0}^{\prime}=0$. If $f_{0}^{\prime}(p)=0$, then once again $y$ divides $f_{0}^{\prime}$, which contradicts the fact that $f_{0}=0$ is reduced. Hence, $f_{0}^{\prime}$ is a unit and $f_{0}$ is nonsingular at $p$.

Continuing to work in the transformed coordinates, we find that $X\left(f_{1}\right)=\xi f_{1}$ on $y=0$, for some $\xi \in \mathbb{C} \llbracket x \rrbracket$, and hence, if $f_{1}(p)=0$, then $y$ must also divide $f_{1}=0$. Transforming back to the original coordinates, and recalling that $f_{0}$ is irreducible, we see that $f_{0}$ must divide $f_{1}$, and hence $F$ is degenerate.

Proposition 3.9. If $F$ is a generalized invariant algebraic curve and $p$ a critical point of $X$ through which $F$ does not pass, then all the terms $L_{i}$ of the cofactor $L_{F}$ must vanish at $p$.

Proof. This is a very simple induction, using (3-4). This result generalizes the one given in Proposition 2.14.

If we are working locally in $\mathbb{C} \llbracket x, y \rrbracket[\varepsilon] /\left(\varepsilon^{k}\right)$, then we can multiply $F$ by a unit $h \in \mathbb{C} \llbracket x, y \rrbracket[\varepsilon] /\left(\varepsilon^{k}\right)$, changing $L_{F}$ to $L_{F}+X(h) / h$ in (3-2). If $F$ is locally nondegenerate (that is, $f_{1}$ is not a multiple of $f_{0}$ locally) and of order $k$, then the same will clearly be true for $h F$.

Proposition 3.10. Suppose that $F$ is a generalized invariant algebraic curve based on a curve $f=0$, which passes through a critical point $p$. If the critical point is a resonant node, then the order of $F$ is at most two. If the critical point is a resonant 
saddle or a saddle-node, then $F$ can have order at most $k+1$, where $k$ is given by the formal normal forms of these points: (2-10) or (2-11). In particular, in these cases we obtain a bound for the multiplicity of the curve $f=0$.

Proof. With an eye toward Proposition 3.13 below, we prove a slightly stronger version of this proposition. We shall relax our assumption that $f_{0}=f$ is irreducible, and merely impose that one of the branches through the critical point has multiplicity one and that $f_{1}$ does not vanish identically on this branch. Without loss of generality, we can assume that the critical point is at the origin and work locally over $\mathbb{C} \llbracket x, y \rrbracket[\varepsilon]$; hence, we can assume that, after a change of coordinates, the vector field is in one of the forms (2-9), (2-10) or (2-11). We consider each case separately to find the maximal value of $n$ such that (3-2) holds modulo $\varepsilon^{n}$.

Resonant node (2-9). In this case, the only possible branch through the origin is $x=0$, and so $f_{0}=x \ell$, where $\ell(0) \neq 0$. We can therefore divide through by $\ell$, assume that $f_{0}=x$, and hence $L_{0}=1$. We multiply $F$ by a suitable unit in $\mathbb{C} \llbracket x, y \rrbracket[\varepsilon]$ to remove all monomials which are multiples of $x$ from the remaining $f_{i}, i>0$. Now $X\left(f_{1}\right)=f_{1}+x L_{1}$, which restricted to $x=0$ gives $k y \partial f_{1} / \partial y=f_{1}$, and hence $f_{1}=c_{1} y^{k}$ for some constant $c_{1}$. This implies that $k=1$, and $c_{1}$ must be nonzero to ensure that $f_{1}$ does not vanish on $x=0$. From (3-2) we find that $L_{1}=c_{1}$. If the curve has multiplicity greater than two, we need to be able to solve $X\left(f_{2}\right)=f_{2}+c_{1}^{2} y+x L_{2}$. However, restricting this equation to $x=0$ we find that there is no $f_{2} \in \mathbb{C} \llbracket y \rrbracket$ which satisfies this equation for $c_{1} \neq 0$. The multiplicity is therefore at most two.

Resonant saddle (2-10). In this case, by symmetry, we can choose $x=0$ to be the branch with multiplicity one, and take $f_{0}=x y^{m} \ell$, with $\ell(0) \neq 0$. We divide through by $\ell$ as before, and assume $f_{0}=x y^{m}$ with

$$
L_{0}=\left(1+a u^{k}\right)-m(p / q)\left(1+(a-1) u^{k}\right) .
$$

Now (3-2) gives

$$
y \frac{\partial f_{1}}{\partial y}=\left(m-\frac{q}{p}\right) f_{1},
$$

on $x=0$, which implies that $f_{1}=c y^{m-q / p}$ on $x=0$ for some constant $c \neq 0$ (since $x$ cannot divide $f_{1}$, by assumption). Thus we need $p=1$ and $m \geq q$.

Now, since $y$ is a (degenerate) invariant algebraic curve of infinite order, by Proposition 3.7 we can divide $F$ by any power of $y$ common to all the $f_{i}$, to leave a generalized invariant algebraic curve of the same order as $F$. Therefore, we can assume without loss of generality that all factors $y$ of $F$ have been removed, and take $f_{r}$ to be the first of the $f_{i}$ which is not divisible by $y$. Then (3-2) implies that

$$
x \frac{\partial f_{r}}{\partial x}=\left(1-m \frac{p}{q}\right) f_{r}
$$


on $y=0$. As before, we need $1-m p / q$ to be a nonnegative integer, and this implies that $q$ divides $m$; since $m \geq q$, we see that $m=q$. However, this implies that $f_{0}=x y^{q}$; from the previous discussion, $f_{1}=c$ on $x=0$, and $L_{0}=u^{k}$.

Now, on $y=0$ we have

$$
x \frac{\partial f_{1}}{\partial x}=\left(1-m \frac{p}{q}\right) f_{1},
$$

so $f_{1}$ is just a constant on $y=0$, and hence $f_{1}=c \bmod (x y)$. Let $h(x) y^{r}, r>0$, be the first nonzero term in the expansion of $f_{1}-c$ in terms of $y$. If $r<q=m$, then we have

$$
x \frac{\partial h}{\partial x}=(1-(m-r)(p / q)) h,
$$

which is impossible. Thus, $f_{1}=c \bmod \left(x y^{q}\right)$, and, by multiplying $F$ by some suitable unit in $\mathbb{C} \llbracket x, y \rrbracket[\varepsilon]$, we can take $f_{1}=c \neq 0$ and $L_{1}=-c u^{k-1}$.

Now, by induction, we can show from (3-4) with $i=0, \ldots, k$ that all the other $f_{i}$ for $i=2, \ldots, k$ can also be chosen to be constants after multiplication by a suitable unit, and that $L_{i}=(-c)^{i} u^{k-i}+O\left(u^{k-i+1}\right)$. However, when $i=k+1$, it is not possible to satisfy (3-4), as the right hand side has a constant term $(-c)^{k}$, and hence the multiplicity is at most $k+1$.

Saddle-node (2-11). In this case we operate similarly to the saddle case. Let $f_{0}=$ $x^{r} y^{s} \ell$, and divide $F$ through by $\ell$ as before; we have $L_{0}=r+(s+a r) y^{k}$. Let $f_{t}$ be the first of the $f_{i}$ which is not divisible by $x$ (as above, we assume without loss of generality that a power of $x$ common to all the $f_{i}$ has been removed), then (3-2) on $x=0$ implies that

$$
y^{k+1} \frac{\partial f_{t}}{\partial y}=\left(r+(s+a r) y^{k}\right) f_{t} .
$$

Thus, we have $r=0$ and hence $s=1$ (as, by hypothesis, the branch must be reduced) which gives $L_{0}=y^{k}$. Since $f_{0}=y$, we can multiply $F$ by a suitable unit in $\mathbb{C} \llbracket x, y \rrbracket[\varepsilon]$ so that all the $f_{i}, i>0$, are just functions of $x$ alone. On $y=0$, (3-2) gives

$$
x \frac{\partial f_{1}}{\partial x}=r f_{1}=0
$$

and hence $f_{1}=c$ on $x=0$ for some constant $c \neq 0$ (by the nondegeneracy assumption). Let $F^{\prime}$ be result of setting $x=0$ in $F$. It is clear that $F^{\prime}$ still satisfies (3-2) up to the same order in $\varepsilon$ as $F$, if we also substitute $x=0$ in the cofactor. Thus, $F^{\prime}$ defines a nondegenerate generalized invariant algebroid curve of at least the same order as $F$. Hence, we can assume that all the $f_{i}$ are in fact constants. It is easy now to show by induction that the $L_{i}=(-c)^{i} y^{k-i}+O\left(y^{k-i+1}\right)$ for $i>0$, and that (3-2) can only be satisfied up to terms in $\varepsilon^{k}$.

Remark 3.11. Note that in these three cases, if $f=0$ is irreducible, we have shown that, after a change of coordinates in $\mathbb{C} \llbracket x, y \rrbracket$, the generalized algebraic curve $F$ 
factorizes as $G F_{0}$ over $\mathbb{C} \llbracket x, y \rrbracket[\varepsilon] /\left(\varepsilon^{k}\right)$, where $G$ is a unit in $\mathbb{C} \llbracket x, y \rrbracket[\varepsilon] /\left(\varepsilon^{k}\right)$, and $F_{0}$ is given by:

- $F_{0}=x+c y+O\left(\varepsilon^{2}\right), c \neq 0$, for a node;

- $F_{0}=x y+c \varepsilon+O\left(\varepsilon^{2}\right), c \neq 0$, for a resonant saddle;

- $F_{0}=y+(c+O(x)) \varepsilon+O\left(\varepsilon^{2}\right), c \neq 0$, for a saddle-node.

Furthermore, it is clear from Proposition 3.8 that, away from a critical point on $f=0$, we can also factorize $F$ after a change of coordinates in $\mathbb{C} \llbracket x, y \rrbracket$ to get $F=G F_{0}$ with

- $F_{0}=y+(c+O(x)) \varepsilon+O\left(\varepsilon^{2}\right), c_{1} \neq 0$, for a noncritical point on $f=0$.

The next corollary will justify our choice of conditions imposed in Section 7 in the case of holonomic multiplicity.

Corollary 3.12. Suppose that $f=0$ is a nonsingular curve, and $F$ a generalized algebraic curve based on $f=0$ of order $m>1$. The only elementary critical points through which the curve $F$ can pass are:

- A linearizable critical point with an integral ratio of eigenvalues $n$. When brought to the form (2-8), if the curve corresponds to the line $y=0$, then $\lambda=n$.

- A resonant node with ratio of eigenvalues equal to unity. If one occurs, then $m$ is at most two.

- A saddle node. When brought to the form (2-11), the curve must correspond to the line $y=0$ (the strong manifold of the saddle-node), and $m \leq k+1$.

Proof. Apart from the three cases considered in Theorem 2.15, we only need to consider the case of a linearizable critical point, which is easily deduced from the same type of argument as above.

Proposition 3.13. Let $F$ be a generalized invariant algebraic curve, and $p$ a critical point through which it passes. After a finite number of blow-ups, we can resolve the critical point into a number of elementary singularities. If $p$ is a critical point which lies on the strict transform of the curve $f=0$, then the order of $F$, and hence the multiplicity of $f=0$, is bounded by 2 , if the critical point is a resonant node, and by $k+1$, if the critical point is a resonant saddle or a saddle-node (where $k$ is obtained from the formal normal forms (2-10) and (2-11)).

Proof. The only thing we need to check is that generalized invariant algebraic curves are taken to generalized invariant algebraic curves in each chart of each successive blow-up, which is easily done. The only complication is that, under the blow-up, exceptional divisors will appear in the expression for $F$. However, the 
strict transform of $f=0$ always appears with multiplicity one, so that we can still apply the proof of Proposition 3.10 as before.

\section{Integrable multiplicity}

Integrable multiplicity deals with the number of exponential factors associated to the invariant algebraic curve. In accordance with our policy to consider only the 'generic' case of an irreducible and reduced curve $f=0$, we shall restrict the exponential coefficients we consider to ones whose denominators are just powers of $f$. We also assume that $X$ acts nontrivially on $f=0$ (otherwise, we divide $X$ by an appropriate power of $f$ ).

\section{A. Definition of integrable multiplicity.}

Definition 4.1. We say that an exponential factor associated to an invariant algebraic curve $f=0$ is of order $k$ if it can be written in the form

$$
h=\exp \left(g / f^{k}\right)
$$

where $f$ does not divide $g$ and the degree of $g$ is at most $k$ times the degree of $f$. Any such $f$ and $g$ must obey the equations

$$
X(f)=f L_{f} \quad \text { and } \quad X(g)=k g L_{f}+f^{k} L_{h},
$$

for some polynomial $L_{h}$ of degree at most $d-1$. We call $g / f^{k}$ the exponential coefficient of the exponential factor.

If we have two exponential factors of the same order associated to the same curve, $\exp \left(g / f^{k}\right)$ and $\exp \left(g^{\prime} / f^{k}\right)$ say, then $X\left(g^{\prime} / g\right)=0$ on $f=0$ from (4-1). If $f$ is of degree $n$, then $g$ and $g^{\prime}$ are of degree at most $k n$, by definition. Since $f$ is irreducible and $X$ acts nontrivially on $f=0, g^{\prime} / g$ must be equal to some constant $c$ on $f=0$, and hence $g^{\prime}=c g+f g^{\prime \prime}$ for some polynomial $g^{\prime \prime}$ of degree at most $(k-1) n$. We can therefore replace the second exponential factor by an exponential factor $\exp \left(g_{3} / f^{k-1}\right)$ of order $k-1$. Repeating this process, we can replace any set of exponential factors by ones with strictly increasing orders. In general, there may be gaps in the sequence of the orders of these exponential factors.

Definition 4.2. We shall say that the invariant algebraic curve $f=0$ has integrable multiplicity $m$ with respect to $X$ if $m$ is the largest integer for which the following is true: there are $m-1$ exponential factors $\exp \left(g_{j} / f^{j}\right), j=1, \ldots, m-1$, with $\operatorname{deg} g_{j} \leq j \operatorname{deg} f$, such that each $g_{j}$ is not a multiple of $f$.

If $g / f$ is a rational first integral with $\operatorname{deg} g \leq \operatorname{deg} f$, then the integrable multiplicity is infinite, taking $\exp \left(g^{i} / f^{i}\right)$ as the exponential factors. The converse follows from Theorem 5.3 and the equivalence of integrable and algebraic multiplicity shown in Section 5 below. 
4B. Multiplicity and integrable multiplicity. We show that the integrable multiplicity of a curve is the same as its (infinitesimal) multiplicity. We use the assumption that the curve $f=0$ is irreducible and reduced in an essential way. The generalization of these results, and the ones in the following sections, to reducible curves is still an open and interesting problem.

Theorem 4.3. Let $X$ be a polynomial vector field and $f=0$ an irreducible and reduced invariant algebraic curve. The integrable multiplicity and the (infinitesimal) multiplicity of the curve are the same.

Proof. This follows from Propositions 4.4 and 4.5.

Proposition 4.4. The integrable multiplicity is at least as great as the multiplicity.

Proof. Suppose the vector field $X$ has a nondegenerate invariant algebraic curve of order $k$,

$$
F=f_{0}+\varepsilon f_{1}+\cdots+\varepsilon^{k-1} f_{k-1},
$$

based on $f$; by definition, $f_{0}=f$ and $f_{1} / f_{0}$ is a nonconstant function. Let

$$
L_{F}=L_{0}+\varepsilon L_{1}+\cdots+\varepsilon^{k-1} L_{k-1}
$$

be the cofactor of $F$. A simple calculation shows that the logarithm of $F$ can be written as

$$
\log F=\log f_{0}+\varepsilon \frac{g_{1}}{f_{0}}+\ldots \varepsilon^{k-1} \frac{g_{k-1}}{f_{0}^{k-1}}
$$

for some polynomials $g_{j}$ of degree at most $j \operatorname{deg} f_{0}$.

(Note that our use of logarithmic terms is mainly formal: if we need an analytic expression, we can take any branch of $\log f_{0}$ as an analytic function to represent this; but we will mainly consider $\log f_{0}$ as an element in some appropriate differential field extension of $\mathbb{C}(x, y)$. Similarly, $\log F$ will denote a quantity in $K[\varepsilon] /\left(\varepsilon^{k}\right)$, where $K$ is some differential extension of $\mathbb{C}(x, y)$ satisfying $\delta(\log F)=\delta F / F$, for $\delta=d / d x$ or $d / d y$.)

In addition, the polynomials $g_{j}$ can be expressed as polynomials of the $f_{i}$ for $i=0, \ldots, j$ :

$$
g_{j}=P_{j}\left(f_{0}, \ldots, f_{j}\right)=\frac{(-1)^{j-1}}{j} f_{1}^{j}+f_{0} Q_{j}\left(f_{0}, \ldots, f_{j}\right)
$$

for some polynomials $P_{j}$ and $Q_{j}$. In particular, the polynomials $g_{j}$ and $f_{0}$ are coprime. Now, $X(\log F)=L_{F}$, and it follows directly that the functions $\exp \left(g_{i} / f_{i}\right)$, $i=1, \ldots, k-1$, are exponential factors of $X$ whose cofactors are just the $L_{i}$.

Proposition 4.5. The multiplicity is at least as great as the integrable multiplicity.

Proof. Suppose that there exists polynomials $g_{1}, \ldots, g_{m-1}$ such that $\exp \left(g_{j} / f^{j}\right)$ are exponential factors of order $j$ for $j=1, \ldots, m-1$. We wish to find $f_{i}$, for 
$i=1, \ldots, m-1$ such that $F=f_{0}+\varepsilon f_{1}+\cdots+\varepsilon^{m-1} f_{m-1} \in \mathbb{C}[x, y, \varepsilon] /\left(\varepsilon^{m}\right)$ is a nondegenerate generalized invariant algebraic curve of order $m$ based on $f$.

When $m=2$ the proposition follows directly by taking $f_{1}=g_{1}$. We proceed by induction, supposing that the proposition holds for $m=k$ and wishing to prove the statement for $m=k+1$. In fact, we take a stronger statement as our inductive hypothesis: there exist constants $\alpha_{i j}$ such that

$$
F=\exp \left(\log f_{0}+\varepsilon \frac{\tilde{g}_{1}}{f_{0}}+\ldots \varepsilon^{k-1} \frac{\tilde{g}_{k-1}}{f_{0}^{k-1}}\right)
$$

is a generalized invariant algebraic curve of order $k$, with $\tilde{g}_{i}=\sum_{j=1}^{i} \alpha_{i j} f_{0}^{i-j} g_{j}$ and $\alpha_{i i} \neq 0$ for all $0<i<k$.

Without loss of generality, by the inductive hypothesis we can replace the $g_{j}$ by the $\tilde{g}_{j}, j=1, \ldots, k-1$, as this takes exponential factors to exponential factors of the same order. Thus, there are polynomials $f_{i}, i=0, \ldots, k-1$, of degree at most $n$ satisfying (3-4) for some polynomials $L_{i}$; the $g_{i}$ are given by (4-3).

Suppose now that we have an exponential factor $\exp \left(g_{k} / f^{k}\right)$ with $g_{k}$ coprime to $f$. We set

$$
F^{\prime}=f_{0}+\varepsilon f_{1}+\cdots+\varepsilon^{k-1} f_{k-1},
$$

which satisfies $X\left(F^{\prime}\right)=F^{\prime} L_{F^{\prime}} \bmod \varepsilon^{k+1}$, with

$$
L_{F^{\prime}}=L_{0}+\varepsilon L_{1}+\cdots+\varepsilon^{k-1} L_{k-1}+\varepsilon^{k} L_{k}^{\prime} \quad \text { and } \quad L_{k}^{\prime}=-\sum_{i=1}^{k-1} \frac{f_{i} L_{k-i}}{f_{0}} .
$$

We calculate the logarithm of $F^{\prime}$, as before, to get

$$
\log F^{\prime}=\log f_{0}+\varepsilon \frac{g_{1}}{f_{0}}+\ldots \varepsilon^{k-1} \frac{g_{k-1}}{f_{0}^{k-1}}+\varepsilon^{k} \frac{g_{k}^{\prime}}{f_{0}^{k}},
$$

for some polynomial $g_{k}^{\prime}$ of order at most $k \operatorname{deg} f_{0}$, which must satisfy

$$
X\left(g_{k}^{\prime}\right)=k L_{0} g_{k}^{\prime}+f_{0}^{k} L_{k}^{\prime}=k L_{0} g_{k}^{\prime}-f_{0}^{k-1} \sum_{i=1}^{k-1} f_{i} L_{k-i} .
$$

From (4-2) we know that $g_{k}^{\prime}=(-1)^{k-1} f_{1}^{k} / k$ on $f_{0}=0$, and hence $X\left(g_{k} / f_{1}^{k}\right)=0$ on $f_{0}=0$. It follows that $g_{k}=c_{k} f_{1}^{k}$ on $f_{0}=0$ for some constant $c_{k}$. By hypothesis, $c_{k} \neq 0$, otherwise $f$ would divide $g_{k}$. Thus, $g_{k}=c_{k}(-1)^{k-1} k\left(g_{k}^{\prime}+f_{0} g_{k-1}^{\prime}\right)$ for some polynomial $g_{k-1}^{\prime}$ of degree at most $(k-1) d$. We can then show that

$$
X\left(g_{k-1}^{\prime}\right)=(k-1) L_{0} g_{k-1}^{\prime}+f_{0}^{k-2}\left(\sum_{i=1}^{k-1} f_{i} L_{k-i}+(-1)^{k-1} f_{0} M_{k} / c_{k} k\right),
$$

where $M_{k}$ is the cofactor of $\exp \left(g_{k} / f^{k}\right)$. 
Now, we find that $X\left(g_{k-1}^{\prime} / f_{1}^{k-1}\right)=0$ on $f_{0}=0$, and so

$$
g_{k-1}^{\prime}=c_{k-1}(-1)^{k-2}(k-1) g_{k-1}+f_{0} g_{k-2}^{\prime}
$$

for some constant $c_{k-1}$, where $g_{k-2}^{\prime}$ is a polynomial of degree at most $(k-2) d$ which satisfies

$$
\begin{aligned}
& X\left(g_{k-2}^{\prime}\right)=(k-2) L_{0} g_{k-2}^{\prime} \\
& +f_{0}^{k-3}\left(\sum_{i=1}^{k-1} f_{i} L_{k-i}+(-1)^{k-1} f_{0} M_{k} / c_{k} k-c_{k-1}(-1)^{k-2}(k-1) f_{0} L_{k-1}\right) .
\end{aligned}
$$

It is clear that we can repeat this last step until we get to $g_{1}^{\prime}$ of degree $d$ satisfying

$$
\begin{aligned}
& X\left(g_{1}^{\prime}\right)=L_{0} g_{1}^{\prime}+\sum_{i=1}^{k-1} f_{i} L_{k-i} \\
& \quad+f_{0}\left((-1)^{k-1} M_{k} / c_{k} k-c_{k-1}(-1)^{k-2}(k-1) L_{k-1}-\cdots-c_{2}(-1) 2 L_{2}\right) .
\end{aligned}
$$

Thus, we can take $f_{k}=g_{1}^{\prime}$ to get a generalized invariant algebraic curve

$$
\tilde{F}=f_{0}+\varepsilon f_{1}+\cdots+\varepsilon^{k} f_{k}
$$

of order $k+1$, taking

$$
L_{k}=(-1)^{k-1} M_{k} / c_{k} k-c_{k-1}(-1)^{k-2}(k-1) L_{k-1}-\cdots-c_{2}(-1) 2 L_{2}
$$

in the cofactor. The proof follows by induction and a chase through the defining equations for the $g_{i}^{\prime}$.

Remark 4.6. It follows directly from this proof that the span of the cofactors of the exponential factors is equal to the span of the $L_{i}$ of the generalized curve $F$.

\section{Algebraic multiplicity}

The definitions and results of this section come from [Pereira 2001]. We state and prove simplified versions adapted to the complex plane. In fact, the notion of extactic curves already appears in the work of Lagutinskii (for more details see [Dobrovol'skii et al. 1998]).

5A. Definition. As usual, we assume that the vector field $X$ is reduced.

Definition 5.1. If $X$ is a vector field on $\mathbb{C}^{2}$, the $n$-th extactic curve of $X, \mathscr{E}_{n}(X)$, is given by the equation

$$
\operatorname{det}\left(\begin{array}{cccc}
v_{1} & v_{2} & \ldots & v_{l} \\
X\left(v_{1}\right) & X\left(v_{2}\right) & \ldots & X\left(v_{l}\right) \\
\vdots & \vdots & \ldots & \vdots \\
X^{l-1}\left(v_{1}\right) & X^{l-1}\left(v_{2}\right) & \ldots & X^{l-1}\left(v_{l}\right)
\end{array}\right)
$$


where $v_{1}, v_{2}, \ldots, v_{l}$ is a basis of $\mathbb{C}_{n}[x, y]$, the $\mathbb{C}$-vector space of polynomials in $\mathbb{C}[x, y]$ of degree at most $n$, and we take $l=(n+1)(n+2) / 2, X^{0}\left(v_{i}\right)=v_{i}$, and $X^{j}\left(v_{i}\right)=X^{j-1}\left(X\left(v_{i}\right)\right)$. A point where $\mathscr{E}_{n}(X)=0$ is called an $n$-inflection point of the vector field $X$.

Observe that the definition of an extactic curve is independent of the chosen basis of $\mathbb{C}_{n}[x, y]$. A 1-inflection point is either a critical point of the vector field or the trajectory at that point has an inflection point in the usual sense, that is, it has a triple intersection point with some line. In general, given a trajectory of a generic vector field at a nonsingular point, we can find a curve of degree $n$ which will have an $n(n+3) / 2$-fold intersection with the trajectory at that point (that is, the number of effective parameters of a general plane curve of degree $n$ ). An $n$-inflection point is either a critical point of the vector field or a point where the vector field has an tangency with a curve of degree $n$ at that point which is greater than $n(n+3) / 2$. This can be clearly seen if we consider the Taylor series expansion for the time evolution of a function of $x$ and $y$ along the trajectory starting at the point $\left(x_{0}, y_{0}\right)$ :

$$
F(x(t), y(t))=F\left(x_{0}, y_{0}\right)+\left.X(F)\right|_{\left(x_{0}, y_{0}\right)} t+\left.X^{2}(F)\right|_{\left(x_{0}, y_{0}\right)} t^{2} / 2+\ldots
$$

In our case, the function $F$ is a curve of degree $n$, and hence a linear combination of the first row of the extactic matrix at the point $\left(x_{0}, y_{0}\right)$. In fact, it is clear that the other coefficients of the Taylor series (5-2) are given by the same linear combination of the other rows of the extactic matrix at $\left(x_{0}, y_{0}\right)$. Thus, the determinant of the extactic matrix vanishes at $\left(x_{0}, y_{0}\right)$ if and only if we can find a linear combination of its columns which is zero, and this is the same as finding a linear combination of monomials of degree $n$ or less for which the first $(n+1)(n+2) / 2$ terms in the series (5-2) vanish.

Proposition 5.2. Every algebraic curve of degree $n$ invariant by the vector field $X$ is a factor of $\mathscr{E}_{n}(X)$.

Proof. Let $f$ be an invariant algebraic curve of degree $n$. As it was observed, the choice of the basis of the $\mathbb{C}$-vector space plays no role in the definitions of extactic curve, and therefore we can take $v_{1}=f$. Since $X^{i}(f)$ is divisible by $f$ for all $i$, it is clear that $f$ must be a factor of $\mathscr{E}_{n}(X)$, since it divides every element in the first column of its corresponding matrix.

Theorem 5.3. Let $X$ be a vector field on $\mathbb{C}^{2}$. We have $\mathscr{E}_{n}(X)=0$ and $\mathscr{E}_{n-1}(X) \neq 0$ if and only if $X$ admits a rational first integral of exact degree $n$.

Proof. The theorem will follow from the simpler statement that the vanishing of $\mathscr{E}_{n}(X)$ is equivalent to the existence of a rational first integral of degree at most $n$. Clearly, if such a first integral exists, then the level curves of the first integral are just curves of degree at most $n$, and hence invariant. By Proposition 5.2, these 
must factor out of the extactic $\mathscr{E}_{n}(X)$. Since there are an infinite number of these curves, we have $\mathscr{E}_{n}(X) \equiv 0$.

Conversely, if $\mathscr{E}_{n}(X) \equiv 0$, then the rows of the extactic matrix are linearly dependent, and hence there are rational functions $\alpha_{i} \in \mathbb{C}(x, y)$ such that

$$
\mathcal{N}_{j}:=\sum_{i=1}^{k} \alpha_{i} X^{j}\left(v_{i}\right)=0, \quad j=0, \ldots, k-1,
$$

when $k=(n+1)(n+2) / 2$. Now, take $k$ to be the smallest value such that there exist rational functions $\alpha_{i}$ for $i=1, \ldots, k$, not all zero, and $v_{i} \in \mathbb{C}_{n}[x, y]$ for $i=1, \ldots, k$ linearly independent over $\mathbb{C}$, such that (5-3) holds. From the discussion above, we must have $k \leq(n+1)(n+2) / 2$, and it is also clear that $k>1$. Without loss of generality, we can also assume that $\alpha_{k}=1$.

Now,

$$
X\left(\mathcal{N}_{j}\right)-\mathcal{N}_{j+1}=\sum_{i=1}^{k} X\left(\alpha_{i}\right) X^{j}\left(v_{i}\right)=0, \quad j=0, \ldots, k-2,
$$

and so, from the minimality of $k$, we see that the terms $X\left(\alpha_{i}\right)$ must all vanish. Hence, each of the $\alpha_{i}$ are either rational first integrals or constants. However, if all the $\alpha_{i}$ are constants, then $\mathcal{N}_{0}=0$ is a nontrivial relationship on $x$ and $y$, which is not possible. Thus, at least one of the $\alpha_{i}$ must give a rational first integral of the vector field $X$.

Finally, we note that, since the $\alpha_{i}$ are constant on each trajectory, with at least one of the $\alpha_{i}$ not identically zero, then for almost all trajectories $\mathcal{N}_{0}$ gives a nontrivial polynomial of degree at most $n$ which vanishes on that trajectory.

Therefore, if $r(x, y) / s(x, y)$ is a rational first integral of minimal degree greater than $n$, then its level curves $r(x, y)-\lambda s(x, y)=0$ must factorize into curves of degree at most $n$ for almost all $\lambda$. By Bertini's second theorem (see for example [Kleiman 1998]), we can find polynomials $u(x, y)$ and $v(x, y)$ such that

$$
r(x, y)-\lambda s(x, y)=\phi_{1}(u(x, y), v(x, y))-\lambda \phi_{2}(u(x, y), v(x, y)),
$$

where $\phi_{1}$ and $\phi_{2}$ are homogeneous nonlinear polynomials of the same degree. Thus,

$$
r / s=\phi_{1}(u / v, 1) / \phi_{2}(u / v, 1),
$$

and it is easy to see that $u / v$ defines a rational first integral of smaller degree than $r / s$. By repeating this argument, we eventually find a rational first integral of degree at most $n$.

Definition 5.4. An invariant algebraic curve $f$ of degree $n$ for the vector field $X$ has algebraic multiplicity $k$ when $k$ is the greatest positive integer such that the $k$-th power of $f$ divides $\mathscr{E}_{n}(X)$. 
Example 5.5. Consider the 2-parameter family of vector fields which appears in [Schlomiuk and Vulpe 2004]:

$$
X_{(t, b)}=\left(2 t^{2}-2 x^{2}\right) \frac{\partial}{\partial x}+\left(b-4 x y-2 t^{3} y^{2}\right) \frac{\partial}{\partial y} .
$$

The extactic curve $\mathscr{E}_{1}(X)$ of $X_{(t, b)}$ is

$$
y(x-t)(x+t)\left(x+t^{3} y-t \sqrt{\frac{2+b t}{2}}\right)\left(x+t^{3} y+t \sqrt{\frac{2+b t}{2}}\right)=y l_{t}^{(1)} l_{t}^{(2)} l_{t}^{(3)} l_{t}^{(4)} .
$$

It follows from Proposition 5.2 that every invariant straight line must be contained in the first extactic curve. It can be easily verified that $y$ is not invariant by any vector field of the form $X_{(t, b)}$ with $b \neq 0$, and that, for every $i \in\{1,2,3,4\}, l_{t}^{(i)}$ is an invariant straight line for $X_{(t, b)}$. When $t=0$, the line $x=0$ therefore has algebraic multiplicity 4 .

The extactic curve for a line in a quadratic vector field has degree at most 5 . However, a computational search shows that there is no nontrivial quadratic vector field with a line of multiplicity 5. Interestingly, the vector field

$$
x(1-x) \frac{\partial}{\partial x}+y(1-y) \frac{\partial}{\partial y}
$$

has 5 invariant lines, which means that there is no nontrivial degeneracy of the family of vector fields with 5 invariant lines so that all the lines coalesce. Of all quadratic vector fields with five lines (counting multiplicity) the only other combination which seems possible is given by the vector field

$$
x(1-x) \frac{\partial}{\partial x}+y(1-2 x) \frac{\partial}{\partial y},
$$

with $x=0$ and $x=1$ of multiplicity two and $y=0$ of multiplicity one.

5B. Multiplicity and algebraic multiplicity. We now want to show that algebraic multiplicity is the same as the infinitesimal multiplicity. The proof is fairly involved, since it is not obvious a priori how to construct a generalized invariant algebraic curve from the extactic. For example, if we consider the line $x=0$ in Example 5.5 when $t=0$, there is a generalized invariant algebraic curve $F=x+\varepsilon+\varepsilon^{2} 2 y / b$ of order four (that is, $f_{3}=0$ in this case).

Theorem 5.6. Let $X$ be a polynomial vector field and $f=0$ an irreducible and reduced invariant algebraic curve. The algebraic multiplicity and the (infinitesimal) multiplicity of the curve are the same.

Proof. This follows from Propositions 5.7, 5.8, and 5.10 below. 
If $S$ is an element of $\mathbb{C}[x, y, \varepsilon]$ or $\mathbb{C}[x, y, \varepsilon] /\left(\varepsilon^{k}\right)$, then we shall use the notation $S_{i}$ or $[S]_{i}$ to denote the coefficient of $\varepsilon^{i}$.

Proposition 5.7. Under the conditions in Theorem 5.6 above, the algebraic multiplicity is at least as great as the multiplicity.

Proof. Let $F$ be a nondegenerate generalized invariant algebraic curve of order $k$ based on $f_{0}=0$, with cofactor $L_{F}$, and let $\left\{v_{1}, \ldots, v_{l}\right\}, l=(n+1)(n+2) / 2$, be a basis for $\mathbb{C}_{n}[x, y]$ such that $v_{1}=f_{0}$. If we express each $f_{i}$ as $a_{i} f_{0}+f_{i}^{\prime}$, where $f_{i}^{\prime}$ lies in the span of the $v_{j}$ for $j>1$, then we can write

$$
F=p(\varepsilon) f_{0}+\sum_{i=1}^{k-1} f_{i}^{\prime} \varepsilon^{i}, \quad p(\varepsilon)=1+\sum_{i=1}^{k-1} a_{i} \varepsilon^{i}
$$

Without loss of generality, we can replace $F$ by $(1 / p(\varepsilon)) F$ and assume that each of the $f_{i}$ lie in the span of the $v_{j}, j>1$.

We define

$$
F^{a}=f_{0}^{k} F^{-1}=f_{0}^{k-1}+\varepsilon f_{0}^{k-2} h_{1}+\cdots+\varepsilon^{k-1} h_{k-1}
$$

for some polynomials $h_{j}$ of degree at most $j \operatorname{deg} f$. Clearly,

We also define

$$
X\left(F^{a}\right)=\left(k L_{0}-L_{F}\right) F^{a} .
$$

$$
S^{r}=F^{a} F^{(r)}, \quad \text { where } F^{(r)}=X^{r}(F) .
$$

Under this notation,

$$
X\left(S^{r}\right)=S^{r+1}+\left(k L_{0}-L_{F}\right) S^{r} .
$$

Therefore, if $f_{0}^{m}$ divides $S^{t}$, then it also divides $S^{t+1}$. Since $S^{0}=F^{a} F=f_{0}^{k}$, we see that $f_{0}^{k}$ divides all $S^{r}, r \geq 0$. As above, we let $S_{k-1}^{r}$ denote the coefficient of $\varepsilon^{k-1}$ in $S^{r}$; then,

$$
S_{k-1}^{r}=F_{k-1}^{a} X^{r}\left(f_{0}\right)+\sum_{i=1}^{k-1} F_{k-i-1}^{a} X^{r}\left(f_{i}\right) .
$$

But a simple calculation from the definition of $F^{a}$ shows that $F_{k-1}^{a}$ can be written as $\left(-f_{1}\right)^{k-1}+f_{0} r_{k-1}$ for some polynomial $r_{k-1}$. In particular, it is not a multiple of $f_{0}$. Now, (5-5) shows that

$$
F_{k-1}^{a} X^{r} f_{0}+\sum_{i=2}^{l} \alpha_{i} X^{r} v_{i} \in f^{k} \mathbb{C}[x, y]
$$

for all $r \geq 0$, where the $\alpha_{i}$ are polynomials derived from $F_{j}^{a}, j=0, \ldots, k-2$.

Finally, we consider the extactic determinant (5-1). If we add $\alpha_{i} / F_{k-1}^{a}$ times the $v_{i}$-column to the $v_{1}$-column for each $i=2, \ldots, l$, then, by (5-6), the first column is divisible by $f^{k}$, which finishes the proof. 
Proposition 5.8. Let $f=0$ be an irreducible invariant algebraic curve of degree $n$ of the polynomial vector field $X$ of degree $d$, with cofactor $L$. If $f$ appears as a multiple factor of $\mathscr{E}_{n}$, then there exists a polynomial $g$ of degree at most $n$, not a multiple of $f$, for which $\exp (g / f)$ is an exponential factor of $X$.

Proof. Let $E_{n}$ represent the matrix from which $\mathscr{E}_{n}$ is obtained. We assume, as always, that $X$ does not act trivially on $f=0$. Let $m_{f}$ represent the ideal $f \mathbb{C}[x, y]$; $X$ descends to a derivation $\delta$ on the ring $O_{f}=\mathbb{C}[x, y] / m_{f}$ and hence to the quotient field $K_{f}$. Let $l=(n+1)(n+2) / 2$, as before.

Consider the matrix $E_{n}$, where we choose the basis $\left\{v_{1}, \ldots, v_{l}\right\}$ of $\mathbb{C}_{n}[x, y]$ so that $v_{1}=f$. We have $X^{r} v_{1}=f(X+L)^{r}$, where we consider $L$ as the operator given by multiplication by $L$ (which therefore does not commute with $X$ ). Thus, $\mathscr{E}_{n}=f \operatorname{det} E_{n}^{\prime}$, where $E_{n}^{\prime}$ is obtained from $E_{n}$ by removing the factor $f$ from the first column.

If there is another factor of $f$ in $\mathscr{E}_{n}$, then we have $\operatorname{det} E_{n}^{\prime}=0$ on $f=0$. We now reduce this condition modulo $m_{f}$ and work over the field $K_{f}$. We have det $\bar{E}_{n}^{\prime}=0$, where the bar represents reduction modulo $m_{f}$.

Since the determinant of $\bar{E}_{n}$ is zero, we have elements $\alpha_{1}, \alpha_{2}, \ldots, \alpha_{l}$ of $K_{f}$, not all zero, such that

$$
\mathscr{A}^{r}:=\alpha_{1}(\delta+\bar{L})^{r} 1+\sum_{i=2}^{l} \alpha_{i} \delta^{r} \bar{v}_{i}=0
$$

for $r=0, \ldots, l-1$.

Lemma 5.9. If $\alpha_{1}=0$, there are no solutions of (5-7) for $r=0, \ldots, l-2$.

Proof. Take $q$ to be the smallest integer such that there exist elements $\alpha_{i_{1}}, \ldots, \alpha_{i_{q}}$ of $K_{f}$, not all zero, with

$$
\mathscr{B}^{r}:=\sum_{j=1}^{q} \alpha_{i_{j}} \delta^{r} \bar{v}_{i_{j}}=0, \quad r=0 \ldots q-1 .
$$

One of the $\alpha_{i_{k}}$ must be nonzero, and, by scaling (5-8), we can assume that $\alpha_{i_{k}}=1$.

It is clear that there are no nontrivial solutions of (5-8) for $q=1$. If $q>1$, we find from (5-8) that

$$
\delta \Re^{r}-\mathscr{B}^{r+1}=\sum_{j=1}^{q} \delta \alpha_{i_{j}} \delta^{r} \bar{v}_{i_{j}}=0, \quad r=0 \ldots q-2 .
$$

and hence all the $\delta \alpha_{i_{j}}$ must vanish by the minimality of $q$ (since $\delta \alpha_{i_{k}}=0$ ). Hence, all the $\alpha_{i_{j}}$ lie in $\mathbb{C}$. However, it is not possible for a linear combination (over $\mathbb{C}$ ) of the $\bar{v}_{i}, i=2, \ldots, l$, to vanish on $f=0$. This is because the corresponding linear combination of the $v_{i}, i=2, \ldots, l$, would give a polynomial of degree $n$ which vanished on $f=0$. However, since $f$ is irreducible as a polynomial and of degree 
$n, g$ must be a constant multiple of $f$, and hence $\left\{f, v_{2}, \ldots, v_{l}\right\}$ would not be a basis for $\mathbb{C}_{n}[x, y]$.

From this proof of Lemma 5.9, we must have an $\alpha_{1} \neq 0$ and elements $\alpha_{2}, \ldots, \alpha_{l}$ of $K_{f}$ such that (5-7) holds. Scaling (5-7), we can assume that $\alpha_{1}=1$ without loss of generality. Clearly, one of the $\alpha_{i}, i>1$, must be nonzero also; we will call this value $\alpha_{k}$. Now, (5-7) implies that

$$
(\delta+\bar{L}) \mathscr{A}^{r}-\mathscr{A}^{r+1}=\sum_{i=2}^{l}\left(\bar{L} \alpha_{i}+\delta \alpha_{i}\right) \delta^{r} \bar{v}_{i}=0,
$$

for $r=0, \ldots l-2$. Thus, from Lemma 5.9, $\bar{L} \alpha_{i}+\delta \alpha_{i}=0$ for each $i>1$. Therefore, $\delta\left(\alpha_{i} / \alpha_{k}\right)=0$ for each $i>1$, which means that $\alpha_{i}=q_{i} \alpha_{k}$ for some constants $q_{i} \in \mathbb{C}$.

Finally, we take $g$ to be the polynomial

$$
g=-\sum_{i=2}^{l} q_{i} v_{i}
$$

and $\bar{g}$ the image of $g$ in $K_{f}$. Since $q_{k}=1, g$ is not a multiple of $f$. The first two equations of (5-7) now give

$$
1-\alpha_{k} \bar{g}=0 \quad \text { and } \quad L-\alpha_{k} \delta \bar{g}=0 .
$$

Thus,

$$
X(g)-g L=f M
$$

for some polynomial $M$ which must be of degree at most $d-1$.

Theorem 5.10. Let $f=0$ be an irreducible invariant algebraic curve of degree $n$ of the polynomial vector field $X$ of degree $d$. If $f$ appears with multiplicity $m$ as a factor of $\mathscr{E}_{n}$, then there exists a nondegenerate generalized invariant algebraic curve $F$ of order $m$ based on $f$.

Proof. We proceed by induction. Proposition 5.8 proves the case $m=2$. We assume that we have found a nondegenerate generalized invariant algebraic curve of order $k$,

$$
F=f_{0}+\varepsilon f_{1}+\cdots+\varepsilon^{k-1} f_{k-1},
$$

with $f_{1}$ not a multiple of $f_{0}$ and with cofactor

$$
L_{F}=L_{0}+\varepsilon L_{1}+\cdots+\varepsilon^{k-1} L_{k-1},
$$

and try to construct one of order $k+1$ if $f^{k+1}$ divides $\mathscr{E}_{n}$. We follow the process described in the first paragraph of the proof of Proposition 5.7, take a basis 
$\left\{f, v_{2}, \ldots, v_{l}\right\}$ for $\mathbb{C}_{n}[x, y]$ and divide $F$ by an appropriate unit in $\mathbb{C}[\varepsilon] /\left(\varepsilon^{k}\right)$ so that, without loss of generality, we can assume that

$$
F=f_{0}+\sum_{i=2}^{l} \varepsilon q^{i}(\varepsilon) v_{i}
$$

for some polynomials

and hence,

$$
q^{i}=\sum_{j=0}^{k-2} q_{j}^{i} \varepsilon^{j}
$$

$$
f_{j}=\sum_{i=2}^{l} q_{j-1}^{i} v_{i}, \quad j=1, \ldots, k-1 .
$$

As in Proposition 5.8, we want to work over $K_{f}$ (or, more generally, $K_{f}[\varepsilon]$ ). To simplify notation, any calculations taken over $K_{f}[\varepsilon]$ rather than $\mathbb{C}[x, y]$ will be denoted by " $\bmod \left(f_{0}\right)$ ", and we shall drop the bars employed previously.

We define $\tilde{F}$ by

$$
\tilde{F}=f_{1}+\varepsilon f_{2}+\cdots+\varepsilon^{k-2} f_{k-2} .
$$

It follows from (3-2) that we have

$$
X \tilde{F}=\tilde{F} L_{F} \bmod \left(f_{0}, \varepsilon^{k-1}\right) .
$$

We also take

$$
\tilde{M}^{r}=\tilde{F}^{-1} \tilde{F}^{(r)},
$$

which satisfies the equation

$$
X\left(\tilde{M}^{r}\right)=\tilde{M}^{r+1}-L_{F} \tilde{M}^{r} \bmod \left(f_{0}, \varepsilon^{k-1}\right) .
$$

Thus,

$$
\tilde{M}^{0}=1 \quad \text { and } \quad \tilde{M}^{1}=L_{F} \quad \bmod \left(f_{0}, \varepsilon^{k-1}\right) .
$$

Once again, we denote the coefficient of $\varepsilon^{i}$ in some expression $S$ by $S_{i}$ or, for more complex expressions, by $[S]_{i}$. Thus, from (5-14) we get

(5-15) $\tilde{M}_{q}^{r+1}=X \tilde{M}_{q}^{r}+\left(L_{F} \tilde{M}^{r}\right)_{q}=X \tilde{M}_{q}^{r}+L_{0} \tilde{M}_{q}^{r}+\cdots+L_{q} \tilde{M}_{0}^{r} \quad \bmod \left(f_{0}, \varepsilon^{k-1}\right)$, for $q<k-1$.

From the proof of Proposition 5.7, we know that $f_{0}^{k}$ divides $S^{r}$ for each $r$, and so we can define a sequence of polynomials

which satisfy

$$
M^{r}=\frac{1}{f_{0}^{k}} S^{r}=F^{-1} F^{(r)}
$$

$$
X\left(M^{r}\right)=M^{r+1}-L_{F} M^{r}, \quad M^{0}=1, \quad M^{1}=L_{F} .
$$

Comparing (5-16) with (5-14), we see that

$$
M^{r+1}-\tilde{M}^{r+1}=X\left(M^{r}-\tilde{M}^{r}\right)+L_{F}\left(M^{r}-\tilde{M}^{r}\right) \bmod \left(f_{0}, \varepsilon^{k-1}\right),
$$


and hence, by induction,

$$
M^{r}=\tilde{M}^{r} \bmod \left(f_{0}, \varepsilon^{k-1}\right) .
$$

The proof now follows along similar lines to Proposition 5.8. We know that

$$
S_{k-1}^{r}=F_{k-1}^{a} f_{0}^{(r)}+\sum_{i=1}^{k-1} F_{k-i-1}^{a} X^{r}\left(f_{i}\right) .
$$

Since $F_{k-1}^{a}=\left(-f_{1}\right)^{k-1} \bmod \left(f_{0}\right)$, we have $F_{k-1} \in K_{f}^{*}$.

Thus, assuming that the matrix $E_{n}$ is expressed with respect to the basis $\{f$, $\left.v_{2}, \ldots, v_{l}\right\}$, we can post-multiply $E_{n}$ by

$$
\left(\begin{array}{ccccc}
F_{k-1}^{a} & & & & \\
a_{2} & 1 & & 0 & \\
a_{3} & 0 & \ddots & & \\
\vdots & \vdots & \ddots & 1 & \\
a_{l} & 0 & \ldots & 0 & 1
\end{array}\right)
$$

without changing the multiplicity of $f_{0}$ appearing in its determinant. We call this new matrix $E_{n}^{\prime}$. If we take

$$
a_{i}=\sum_{j=1}^{k-1} F_{k-j-1}^{a} q_{j-1}^{i},
$$

then it is clear from (5-18) that the first column of $E_{n}^{\prime}$ is given by $S_{r}^{k-1}$ while the other columns remain the same. If $f_{0}^{k+1}$ divides $\mathscr{E}_{n}$, then it must also divide $\operatorname{det} E_{n}^{\prime}$. However, there is a factor $f_{0}^{k}$ in every term in the first column of $E_{n}^{\prime}$. We therefore divide this first column by $f_{0}^{k}$ and get a new matrix $E_{n}^{\prime \prime}$, whose first column is given by the elements $M_{k-1}^{r}$ and which has at least one factor of $f_{0}$ dividing its determinant.

Working over $K_{f}$, since the determinant of $E_{n}^{\prime \prime}$ is zero, we can find nonzero elements $\alpha_{i}$ in $K_{f}$, for $i=1, \ldots, l$, such that

$$
\mathscr{C}^{r}:=\alpha_{1} M_{k-1}^{r}+\sum_{i=2}^{l} \alpha_{i} \delta^{k} v_{i}=0 \quad \bmod \left(f_{0}\right), \quad r=0, \ldots, l-1 .
$$

We know from Lemma 5.9 that $\alpha_{1}$ cannot be zero, and so, without loss of generality, we take it to be 1 .

Now, by differentiating (5-19) we get

$$
\begin{aligned}
\mathscr{C}^{r+1} & -\left(\delta+L_{0}\right) \mathscr{C}^{r} \\
& =L_{1} M_{k-2}^{r}+L_{2} M_{k-3}^{r}+\ldots L_{k-1} M_{0}^{r}-\sum_{i=2}^{l}\left(\delta \alpha_{i}+L_{0} \alpha_{i}\right) \delta^{k} v_{i}=0 \quad \bmod \left(f_{0}\right),
\end{aligned}
$$


for $r=0, \ldots, l-2$. However, $M_{i}^{r}=\tilde{M}_{i}^{r} \bmod \left(f_{0}\right)$ for $i<k-1$, and so

$$
f_{1}\left(L_{1} \tilde{M}_{k-2}^{r}+L_{2} \tilde{M}_{k-3}^{r}+\ldots L_{k-1} \tilde{M}_{0}^{r}\right)-\sum_{i=2}^{l} \delta\left(f_{1} \alpha_{i}\right) \delta^{k} v_{i}=0 \bmod \left(f_{0}\right)
$$

for $r=0, \ldots, l-2$. The first term in this expression can be seen to be

$$
f_{1}\left[\left(L_{F}-L_{0}\right) \tilde{M}^{r}\right]_{k-1}
$$

Now, from (5-12), $\tilde{F}=\sum_{i=2}^{l} q^{i}(\varepsilon) v_{i} \bmod \left(f_{0}\right)$, and so

$$
\begin{aligned}
\sum_{i=2}^{l} \delta\left(f_{1} \alpha_{i}\right) \delta^{r} v_{i} & =f_{1}\left[\left(L_{F}-L_{0}\right) \tilde{M}^{r}\right]_{k-1} \\
& =\left[\left(L_{F}-L_{0}\right) \tilde{F}^{-1} f_{1} \sum_{i=2}^{l} q^{i}(\varepsilon) \delta^{r} v_{i}\right]_{k-1} \bmod \left(f_{0}\right),
\end{aligned}
$$

which gives

$$
\sum_{i=2}^{l}\left(\delta\left(f_{1} \alpha_{i}\right)-\left[\left(L_{F}-L_{0}\right) \tilde{F}^{-1} f_{1} q^{i}(\varepsilon)\right]_{k-1}\right) \delta^{r} v_{i}=0 \bmod \left(f_{0}\right),
$$

for $r=0, \ldots, l-2$. Lemma 5.9 thus gives

$$
\delta\left(f_{1} \alpha_{i}\right)=\left[\left(L_{F}-L_{0}\right) \tilde{F}^{-1} f_{1} q^{i}(\varepsilon)\right]_{k-1} \quad \bmod \left(f_{0}\right), \quad i=2, \ldots, l .
$$

We write $q_{0}^{i}=q^{i}(0)$, and so $\tilde{q}^{i}(\varepsilon)=q_{0}^{i}-q^{i}(\varepsilon)$. From (5-13), we get

$$
\delta\left(\tilde{F}^{-1} f_{1} \tilde{q}^{i}(\varepsilon)\right)=\tilde{F}^{-1} f_{1}\left(L_{0}-L_{F}\right) \tilde{q}^{i}(\varepsilon) \bmod \left(f_{0}\right) .
$$

Thus,

$$
\delta\left(f_{1} \alpha_{i}+\left[\tilde{F}^{-1} f_{1} \tilde{q}^{i}(\varepsilon)\right]_{k-1}\right)=\left[\tilde{F}^{-1} f_{1}\left(L_{0}-L_{F}\right) q_{0}^{i}\right]_{k-1} \quad \bmod \left(f_{0}\right) .
$$

Since, by hypothesis, $F$ is nondegenerate, one of the $q_{0}^{i}$, s must be nonzero. Without loss of generality, we shall assume it to be $q_{0}^{l}$. Then,

$$
\delta\left(f_{1}\left(\alpha_{i}-\frac{q_{0}^{i}}{q_{0}^{l}} \alpha_{l}\right)+\left[\tilde{F}^{-1} f_{1}\left(\tilde{q}^{i}(\varepsilon)-\frac{q_{0}^{i}}{q_{0}^{l}} \tilde{q}^{l}(\varepsilon)\right)\right]_{k-1}\right)=0 \bmod \left(f_{0}\right) .
$$

So, there exist constants $c_{k}^{i}$ such that

$$
f_{1}\left(\alpha_{i}-\frac{q_{0}^{i}}{q_{0}^{l}} \alpha_{l}\right)+\left[\tilde{F}^{-1} f_{1}\left(\tilde{q}^{i}(\varepsilon)-\frac{q_{0}^{i}}{q_{0}^{l}} \tilde{q}^{l}(\varepsilon)\right)\right]_{k-1}=c_{k}^{i} \quad \bmod \left(f_{0}\right) .
$$


We set $f_{k}=-\sum_{i=2}^{l} c_{k}^{i} v_{i}$. Equation (5-19) gives

$$
\begin{aligned}
M_{k-1}^{r} & =-\sum_{i=2}^{l} \alpha_{i} \delta^{r} v_{i} \\
& =\sum_{i=2}^{l}\left(-\frac{c_{k}^{i}}{f_{1}}-\frac{q_{0}^{i}}{q_{0}^{l}} \alpha_{l}+\left[\tilde{F}^{-1}\left(\tilde{q}^{i}(\varepsilon)-\frac{q_{0}^{i}}{q_{0}^{l}} \tilde{q}^{l}(\varepsilon)\right)\right]_{k-1}\right) \delta^{r} v_{i} \\
& =\frac{f_{k}^{(r)}}{f_{1}}-\frac{\alpha_{l}}{q_{0}^{l}} f_{1}^{(r)}+\left[\tilde{F}^{-1}\left(\tilde{F}^{(r)}-\frac{\tilde{q}^{l}(\varepsilon)}{q_{0}^{l}} f_{1}^{(r)}\right)\right]_{k-1} \bmod \left(f_{0}\right),
\end{aligned}
$$

for $r=0, \ldots, l-2$, where $f_{i}^{(r)}=\delta^{r} f_{i}$. Taking the cases $r=0$ and $r=1$ in the previous equation, we get

$$
\begin{aligned}
& 0=\frac{f_{k}}{f_{1}}-\frac{\alpha_{l}}{q_{0}^{l}} f_{1}+\left[\tilde{F}^{-1}\left(\tilde{F}-\frac{\tilde{q}^{l}(\varepsilon)}{q_{0}^{l}} f_{1}\right)\right]_{k-1} \bmod \left(f_{0}\right), \\
& L_{k-1}=\frac{\delta f_{k}}{f_{1}}-\frac{\alpha_{l}}{q_{0}^{l}} L_{0} f_{1} \\
& +\left[\tilde{F}^{-1}\left(L_{F} \tilde{F}-\left[L_{F} \tilde{F}\right]_{k-1} \varepsilon^{k-1}-\frac{\tilde{q}^{l}(\varepsilon)}{q_{0}^{l}} L_{0} f_{1}\right)\right]_{k-1} \bmod \left(f_{0}\right),
\end{aligned}
$$

which we rearrange to give

$$
L_{k-1}=\frac{\delta f_{k}-L_{0} f_{k}}{f_{1}}+\left[L_{F}-L_{0}-\tilde{F}^{-1}\left[L_{F} \tilde{F}\right]_{k-1} \varepsilon^{k-1}\right]_{k-1} \bmod \left(f_{0}\right) .
$$

Thus,

$$
\delta f_{k}=L_{0} f_{k}+L_{1} f_{k-1}+\cdots+L_{k-1} f_{1} \bmod \left(f_{0}\right) .
$$

It follows that there exists a polynomial $L_{m}$, necessarily of degree at most $d-1$, such that

$$
X\left(f_{k}\right)=L_{0} f_{k}+L_{1} f_{k-1}+\cdots+L_{k-1} f_{1}+L_{k} f_{0} .
$$

Thus, it is clear that

$$
F^{\prime}=f_{0}+\varepsilon f_{1}+\varepsilon^{2} f_{2}+\cdots+\varepsilon^{k} f_{k},
$$

is a generalized invariant algebraic curve of order $k+1$. This completes the induction.

\section{Geometric multiplicity}

6A. Definition. Let $X$ be a polynomial vector field of degree $d$, and $f=0$ an invariant algebraic curve of degree $n$ which is irreducible and reduced.

Definition 6.1. An invariant algebraic curve $f=0$ of degree $n$ of the vector field $X$ has geometric multiplicity $m$ if $m$ is the largest integer for which there exists a sequence of vector fields $\left(X_{i}\right)_{i>0}$ of bounded degree, converging to $h X$, for 
some polynomial $h$, not divisible by $f$, such that each $X_{r}$ has $m$ distinct invariant algebraic curves, $f_{r, 1}=0, \ldots, f_{r, m}=0$, of degree at most $n$, which converge to $f=0$ as $r$ goes to infinity. If we set $h=1$ in the definition above, then we say that the curve has strong geometric multiplicity $m$.

By convergence of invariant algebraic curves or vector fields we mean the convergence in the parameter space of the coefficients of their defining polynomials.

The condition that the $f_{r, i}$ are also polynomials of degree $n$ is the same as asking that the curves defined by them tend to the curve $f=0$ alone, and not to a multiple of $f=0$ or to a product $f g=0$ with some other algebraic curve.

Note that the definition of geometric multiplicity a priori allows that it take the value infinity. If this is the case, however, it will follow from the equivalence of geometric and algebraic multiplicity that the vector field $X$ has a rational first integral of degree $n$.

We were not able to answer this interesting question:

Question 6.2. Does the strong geometric multiplicity represent a restriction on the definition of geometric multiplicity?

We shall show that the definition above allows us to find a family of curves parameterized by a continuous variable.

Proposition 6.3. Let $f=0$ be an algebraic curve of degree $n$ invariant by the vector field $X$. If the geometric multiplicity of $f=0$ is $m$, there exists an analytic 1-parameter family of polynomial vector fields $\tilde{X}_{t}$ of fixed degree, with $t \in(-\epsilon, \epsilon)$ and analytic in $t$, such that, for each $t \in(-\epsilon, \epsilon) \backslash\{0\}, \tilde{X}_{t}$ has $m$ distinct invariant algebraic curves $\tilde{f}_{t, 1}, \ldots, \tilde{f}_{t, m}$, of degree at most $n$, converging to $f$ as $t$ tends to zero, and $\tilde{X}_{0}=h X$.

Proof. Let $d^{\prime}$ be the maximum degree of the $X_{r}$, and take the parameter space consisting of all the coefficients of a general vector space of degree $d^{\prime}$ together with $m$ general polynomials of degree $n$ (to represent the curves) and $m$ general polynomials of degree $d^{\prime}-1$ (to represent the cofactors). The condition for all $m$ curves to be invariant is given by a number of quadratic relations between the coefficients. We let $\mathscr{L}$ represent the resulting algebraic variety.

The conditions that all the curves are distinct and that the curves and the vector field are nontrivial gives a subvariety $\mathscr{Y}$ of $\mathscr{X}$. The hypothesis of the theorem therefore tells us that the point $p \in \mathscr{Y}$, representing the vector field $X_{0}$ and $m$ copies of the curve $f$, lies in the closure of $\mathscr{X} \backslash \mathscr{Y}$. An infinite number of the $X_{r}$ must lie in at least one irreducible component $\mathscr{L}_{0}$ of $\mathscr{X}$ of dimension $d>0$. We must therefore have $p \in \mathscr{Y}_{0}$, where $\mathscr{Y}_{0}=\mathscr{Y} \cap \overline{\mathscr{X}}_{0}$ has at dimension at most $d-1$. If $d>1$, we can reduce this dimension by restricting our interest to the intersection of $\mathscr{L}_{0}$ with a general hypersurface through $p$. If we repeat this procedure, we end up with a 
variety $V$ passing through $p$ such that $\mathscr{X}_{0} \cap V$ is of dimension one and $\mathscr{Y}_{0}$ is of dimension zero. We can thus find a uniformizing parameter for a neighborhood of $p$ in $\mathscr{X}_{0} \cap V$, and hence an analytic parametrization of the associated vector fields.

In the following sections, we shall show that geometric multiplicity is the same as multiplicity, under the usual assumptions that the algebraic curve $f=0$ is irreducible and reduced. In Section 6B we show that the multiplicity is as least as great as the geometric multiplicity. The final two sections show the converse. In Section $6 \mathrm{C}$ we give a description of all families of polynomial vector fields with prescribed generalized algebraic curves. This is a result of general interest, but will be used in a simple form in the final Section 6D, where we show how to generate a perturbation of the vector field which generates a number of invariant algebraic curves from the curve $f=0$ which is at least as great as the multiplicity of $f=0$. In general we only show that the perturbation is in the class of rational vector fields, but, under some genericity conditions, we show that the perturbation can be chosen to be polynomial, and hence in these cases the integrable multiplicity equals the strong geometric multiplicity.

6B. Multiplicity and geometric multiplicity. We first show that geometric multiplicity is at least as great as the multiplicity. The most direct method is via algebraic multiplicity, using the equivalence established in Section 5B.

Proposition 6.4. The algebraic multiplicity of a curve is at least as great as the geometric multiplicity.

Proof. Suppose that the degree of $f$ is $n$, and let $X_{k}$ be a sequence of vector fields of degree $d^{\prime}$ converging to $X_{0}=h X$, with $m$ distinct invariant algebraic curves $f_{k, 1}, \ldots, f_{k, m}$ converging to $f$. By Proposition 5.2 , the product $f_{k, 1} \ldots f_{k, m}$ must divide $\mathscr{E}_{n}\left(X_{k}\right)$, and so $f^{m}$ divides $\mathscr{E}_{n}\left(X_{0}\right)$ in the limit. But the $(r+1)$-th row of $\mathscr{E}_{n}\left(X_{0}\right)$ is given by

$$
(h X)^{r}\left(v_{1}, \ldots, v_{l}\right)=h^{r} X^{r}\left(v_{1}, \ldots, v_{l}\right)+\sum_{j=0}^{r-1} m_{j}(h) X^{j}\left(v_{1}, \ldots, v_{l}\right)
$$

where $l=(n+1)(n+2) / 2$ and $m_{j}(h)$ is a polynomial in $h$ and its derivatives $X^{i}(h)$ for $i<r$. Thus, the extactic matrix associated to $\mathscr{E}_{n}\left(X_{0}\right)$ can be obtained from the extactic matrix associated to $\mathscr{E}_{n}(X)$ by premultiplying by a lower-triangular matrix with diagonal terms $1, h, h^{2}, \ldots, h^{l-1}$. Taking determinants, we find that

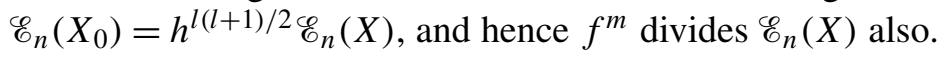

We present an alternative proof of this proposition, which shows in a direct way how exponential factors arise from the coalescence of invariant algebraic curves. We will use this again in Section 8 to discuss the completion of the Darboux theory 
of integrability to limits of families of Darboux first integrals or integrating factors. We use the notation of Proposition 6.3, apart from dropping the tilde over the $X$.

Proposition 6.5. Let $X_{t}, t \in(-\epsilon, \epsilon)$, be an analytic 1-parameter family of vector fields of degree $d$ such that, for each $t \neq 0, X_{t}$ has $m$ distinct invariant algebraic curves $f_{t, i}=0, i=1, \ldots, m$, of degree $n$, varying analytically with $t$ and, as $t$ tends to 0 , converging to an irreducible and reduced curve $f=0$ of degree $n$. There exist analytic functions $\ell_{i, j}(t), i=1, \ldots, m, j=1, \ldots, m-1$, and integers $n_{j}$ such that, as $t$ tends to 0 , the functions

$$
\frac{1}{t^{n_{j}}} \sum_{i=1}^{r} \ell_{i, j} \log f_{t, i}, \quad j=1, \ldots, m-1
$$

tend to exponential coefficients of $X_{0}$ of order $j$.

The equivalent statement in terms of formal power series in $t$ is also true if we replace the idea of a family of invariant algebraic curves by elements $f_{t, i}$ of $\mathbb{C} \llbracket t \rrbracket[x, y]$ satisfying $X\left(f_{t, i}\right)=L_{t, i} f_{t, i}$ for some $L_{t, i} \in \mathbb{C} \llbracket t \rrbracket[x, y]$, and take the logarithms in a formal sense as mentioned before.

Remark 6.6. If the cofactors of the $f_{t, i}$ are denoted by $L_{t, i}$, then the cofactors of the corresponding exponential factors will be given by

$$
L_{j}=\lim _{t \rightarrow 0} \frac{1}{t^{n_{j}}} \sum_{i=1}^{r} \ell_{i, j} L_{t, i}, \quad j=1, \ldots, m-1 .
$$

Choosing $\epsilon$ small enough, the subspace $\sigma_{t} \subset \mathbb{C}_{d-1}[x, y]$ spanned by the cofactors $L_{t, i}$ for $t \neq 0$ is of fixed dimension, say $N$. If we consider the space of $N$-dimensional subspaces of $\mathbb{C}_{d-1}$ (parameterized by the Grassmannian), then the space $\sigma_{0}$ generated by the $L_{j}, j=1, \ldots, m-1$, and the cofactor of $f$ must lie in the limit of the $\sigma_{t}$ as $t$ tends to 0 .

Proof. The proof for the analytic and formal cases are entirely equivalent, so we only present the formal proof. From the hypothesis, there exist polynomials $f_{i}^{(k)} \in$ $\mathbb{C}[x, y]$ of degree at most $n$ such that

$$
f_{t, i}=f+t f_{1}^{(1)}+t^{2} f_{2}^{(2)}+\ldots
$$

We write

$$
\log f_{t, i}=\log f+\sum_{k>0} \frac{g_{i}^{(k)}}{f^{k}},
$$

where the $g_{i}^{(k)}$ are polynomials of degree at most $k n$.

It is sufficient to show that, via (6-1), we can generate $m-1$ linearly independent exponential coefficients of order at most $m-1$, as the conclusion will follow directly from the arguments after Definition 4.1.

Consider the quantity 


$$
M=\frac{\sum_{i=1}^{m} \ell_{i} K_{i} d f_{t, i}}{K},
$$

where

$$
K=\prod_{i=1}^{m} f_{t, i}, \quad K_{i}=\prod_{j \neq i} f_{t, j},
$$

and $\ell_{i} \in \mathbb{C} \llbracket t \rrbracket$, and where $d f_{t, i}$ represents the differential of $f_{t, i}$ with respect to $x$ and $y$ but not $t$. That is, it is an element of $\mathbb{C} \llbracket t \rrbracket \otimes \Omega_{m n}$, where $\Omega_{k}$ is the space of differentials $a(x, y) d x+b(x, y) d y$ with $a$ and $b$ in $\mathbb{C}_{k-1}[x, y]$. Since

$$
M=\sum_{i=1}^{m} \ell_{i} \frac{d f_{t, i}}{f_{t, i}}=d \sum_{i=1}^{n} \ell_{i} \log f_{t, i}=\sum_{i=1}^{n} \ell_{i}\left(\log f+\sum_{k>0} \frac{g_{i}^{(k)}}{f^{k}}\right),
$$

the quantity $M$ is the differential of a function in $\mathbb{C} \llbracket t \rrbracket \otimes \mathbb{C}(x, y)$ if and only if $\sum \ell_{i}=0$.

If $\sum_{i=1}^{m} \ell_{i} K_{i} d f_{t, i}=0$, then, from the fact that $\mathbb{C} \llbracket t \rrbracket[x, y]$ is a unique factorization domain, we see that, if $\ell_{i}$ is nonzero for some $i$, then $f_{t, i}$ divides $d f_{t, i}$. This is impossible, as it implies that $f$ divides $d f$, by setting $t=0$. Thus, the module spanned by the $K_{i} d f_{t, i}$ in $\mathbb{C} \llbracket t \rrbracket \otimes \Omega_{m n}$ is a free module of dimension $m$ over $\mathbb{C} \llbracket t \rrbracket$, and the submodule of those elements that are of the form $\sum_{i=1}^{m} \ell_{i} K_{i} d f_{t, i}$ with $\sum_{i=1}^{m} \ell_{i}=0$ form a free submodule of dimension $m-1$.

Suppose we can choose a basis $\left\{v_{1}, \ldots, v_{m-1}\right\}$ for this latter space such that, when the $v_{i}$ are expressed in the form

$$
v_{i}=t^{n_{i}} v_{i, 0}+O\left(t^{n_{i}+1}\right),
$$

the $v_{i, 0}$ are linearly independent over $\mathbb{C}$. Then there exist series $\ell_{i, j} \in \mathbb{C} \llbracket t \rrbracket$ with $\sum_{i=1}^{m} \ell_{i, j}=0$ such that

$$
\frac{1}{t^{n_{j}}} \frac{v_{j}}{K}=\frac{\sum_{i=1}^{m} \ell_{i, j} K_{i} d f_{t, i}}{K},
$$

whose value at $t=0$ is $v_{j, 0} / f^{m}$. By our choice of space, this element is the differential of a rational function in $\mathbb{C}(x, y)$, and it is clear this must be of the form $w_{j} / f^{m-1}$ for some polynomial $w_{j} \in \mathbb{C}[x, y]$ of degree at most $(m-1) n$. It is also clear that the $w_{j}$ must be linearly independent, since the $v_{j, 0}$ are, and that they must be exponential factors, since

$$
X\left(\frac{w_{j, 0}}{f^{m-1}}\right)=X\left(\lim _{t \rightarrow 0} \frac{1}{t^{n_{j}}} \sum_{i=1}^{r} \ell_{i, j} \log f_{t, i}\right)=\lim _{t \rightarrow 0} \frac{1}{t^{n_{j}}} \sum_{i=1}^{r} \ell_{i, j} L_{t, i} .
$$

It remains to show that the choice of basis can be made. However, this is not hard to achieve. At each step, we order the $v_{i}$ according to their respective values 
of $n_{i}$. If $v_{k, 0}$ depends linearly on $v_{i, 0}$ for $i<k$, then we have

$$
v_{k, 0}=\sum_{i<k} m_{i} v_{i, 0}
$$

for some constants $m_{i} \in \mathbb{C}$, and we can replace $v_{k}$ by

$$
v_{k}-\sum_{i<k} m_{i} v_{i} t^{n_{k}-n_{i}}
$$

Thus, at each step of the process we increases the value of $\sum_{i=1}^{m} n_{i}$. If we can show that this sum is bounded above, then we can only repeat this process a finite number of times until we find a basis with the desired property.

Now, take a basis $\left\{r_{1}, \ldots, r_{p}\right\}$ of $\Omega_{m n}$ and consider the $(m-1) \times p$ matrix whose $(i, j)$-th entry is the coefficient of $r_{j}$ in $v_{i}$ (an element of $\mathbb{C} \llbracket t \rrbracket$ ). The determinants of the $(m-1) \times(m-1)$ minors of this matrix are not all zero (since the $v_{i}$ are linearly independent); so, we can choose one nonzero determinant and write it in the form $c t^{N}+O\left(t^{N+1}\right)$, for some integer $N$ and nonzero constant $c \in \mathbb{C}$. Clearly, $N$ is an upper bound for $\sum_{i=1}^{m} n_{i}$ and, furthermore, the reduction process above does not alter this value of $N$. Hence, our process must terminate after a finite number of steps.

Example 6.7. Consider the 2-parameter family $X_{(t, b)}$ given in Example 5.5. For $t \neq 0$, the vector field has exactly four invariant straight lines $l_{t, i}$, for $i=1, \ldots, 4$. We compute

$$
\begin{aligned}
& \lim _{t \rightarrow 0} \frac{\log l_{t, 2}-\log l_{t, 1}}{t}=\frac{2}{x}, \\
& \lim _{t \rightarrow 0} \frac{\log l_{t, 4}+\log l_{t, 3}-\log l_{t, 2}-\log l_{t, 1}}{t^{3}}=\frac{4 x y-b}{2 x^{2}}, \\
& \lim _{t \rightarrow 0} \frac{l_{t, 4}-l_{t, 3}+\left(b^{2} t^{2} / 32-b t / 4-1\right)\left(\log l_{t, 2}-\log l_{t, 1}\right)}{t^{4}}=\frac{3 b^{3} x^{2}-384 x y+64 b}{192 x^{3}},
\end{aligned}
$$

giving us, when $t=0$, three exponential factors

$$
\exp \frac{2}{x}, \quad \exp \frac{4 x y-b}{2 x^{2}}, \quad \text { and } \quad \exp \frac{3 b^{3} x^{2}-384 x y+64 b}{192 x^{3}},
$$

in addition to the invariant curve $x=0$.

6C. Polynomial systems with prescribed invariant algebraic curves. In this section we want to study the set of vector fields which have a fixed set of generalized algebraic curves. The results given in this section generalize the results in [Christopher et al. 2002] and bear similarities with the work of Walcher [2000] for vector fields with one invariant curve. 
Given a generalized invariant algebraic curve $F$ of order $m$, we can realize it concretely in the space of $m \times m$ matrices by taking

$$
\varepsilon=\left(\begin{array}{ccccc}
0 & 1 & 0 & \ldots & 0 \\
& 0 & 1 & & \vdots \\
& & \ddots & 1 & 0 \\
& 0 & & 0 & 1 \\
& & & & 0
\end{array}\right)
$$

so that

$$
F=f_{0}+f_{1} \varepsilon+\cdots+f_{m-1} \varepsilon^{m-1}=\left(\begin{array}{ccccc}
f_{0} & f_{1} & \ldots & \ldots & f_{m-1} \\
& f_{0} & \ddots & & \vdots \\
& & \ddots & \ddots & \vdots \\
& 0 & & f_{0} & f_{1} \\
& & & & f_{0}
\end{array}\right) .
$$

In the same way we can define $L_{F}$ as a matrix, and (3-2) becomes a matrix equation. It turns out that this concrete realization of $F$ will give a very convenient notation for the work in this section.

The next lemma shows how to 'unfold' $F$ as a matrix.

Lemma 6.8. Given a generalized invariant algebraic curve $F$, considered as a matrix (6-2), we form the polynomials

$$
\tilde{f_{j}}=\sum_{k=0}^{j} \frac{j !}{(j-k) !} f_{k} \eta^{k},
$$

where $\eta$ is an independent variable, and define the matrix $A=\exp (\varepsilon / \eta)$. If we set

$$
\tilde{F}=\operatorname{diag}\left(\tilde{f}_{m-1}, \ldots, \tilde{f}_{0}\right),
$$

then the upper-triangular matrix $F^{\prime}:=A^{-1} \tilde{F} A=\left(b_{i, j}\right)$ is given by

$$
b_{i, j}=\frac{(m-j) !}{(m-i) !(j-i) !}\left(\frac{d}{d \eta}\right)^{j-i} \tilde{f}_{m-i} .
$$

In particular, as $\eta$ tends to zero, $F^{\prime}$ tends to $F$.

Proof. We first show that the $b_{i, j}$ satisfy the equation

$$
b_{i, j}-b_{i+1, j+1}=(j-i+1) \eta b_{i, j+1}, \quad b_{i, i}=\tilde{f}_{0} .
$$

From the expansion of $A=\exp (\varepsilon / \eta)$, we have

$$
F^{\prime}=\sum_{i, j} \frac{1}{i !} \frac{1}{j !} \varepsilon^{i} \tilde{F} \varepsilon^{j} \frac{(-1)^{i}}{\eta^{i+j}}=\sum_{k} B_{k},
$$


where

$$
B_{k}=\sum_{j+i=k} \frac{1}{i !} \frac{1}{j !} \varepsilon^{i} \tilde{F} \varepsilon^{j} \frac{(-1)^{i}}{\eta^{i+j}} .
$$

A simple rearrangement of this formula gives

$$
B_{k} \varepsilon-\varepsilon B_{k}=(k+1) B_{k+1} \eta .
$$

Since post-multiplying by $\varepsilon$ has the effect of shifting the entries of a matrix to the right by one column, while premultiplying by $\varepsilon$ moves all the entries up by one row, we see that $B_{k}$ is just the terms in the $k$-th diagonal of $F^{\prime}$, and the equation (6-5) follows directly from (6-6).

Now we show by induction that the $b_{i, j}$ are given by (6-4). Clearly, this is true when $i=j$. Furthermore, after some manipulation, the right-hand sides of (6-4) satisfy the recurrence relations (6-5), as the $b_{i, j}$ - and so the two expressions for $b_{i, j}$ - must coincide.

Again, given a generalized invariant algebraic curve as above, we define

$$
|F|=\operatorname{det} F=f_{0}^{m} \quad \text { and } \quad F_{i}=[\log F]_{i}
$$

(and not $F_{i}=f_{i}$ ). Thus, $F_{0}=\log f_{0}$ and $F_{i}$ is rational for $i>0$. We also let $\{A, B\}$ denote the Poisson bracket of $A$ and $B$; that is,

$$
\{A, B\}=\frac{\partial A}{\partial x} \frac{\partial B}{\partial y}-\frac{\partial A}{\partial y} \frac{\partial B}{\partial x} .
$$

We shall denote the vector field $\{f, \cdot\}$ by $X_{f}$. Since the denominator of $F_{i}$ for $i>0$ is at worst $f_{0}^{i},|F| X_{F_{i}}=|F|\left\{F_{i}, \cdot\right\}$ is a polynomial vector field. For $i=0$ it is just the vector field $f_{0}^{m-1} X_{f_{0}}$.

Where no confusion can result, the notation $S_{x}$ will mean the derivative of $S$ with respect to $x$. If applied to a vector, it denotes taking the derivatives componentwise.

We will use the previous Lemma 6.8 to show that $|F|\left\{F_{s}, F_{t}\right\}$ is also a polynomial for each pair of integers $r$ and $s$. We define the matrices

$$
B=\left(f_{m-1}, \ldots, f_{0}\right)^{T} \quad \text { and } \quad B^{\prime}=\left(F_{m-1}, \ldots, F_{0}\right)^{T},
$$

and also the matrices

$$
D=\left(\left\{f_{m-i}, f_{m-j}\right\}\right) \quad \text { and } \quad D^{\prime}=\left(\left\{F_{m-i}, F_{m-j}\right\}\right) .
$$

Then $B_{x}^{\prime}=F^{-1} B_{x}$ (this follows directly from post-multiplying by $(0, \ldots, 0,1)^{T}$ the identity $\left.\log (F)_{x}=F^{-1} F_{x}\right)$ and $D^{\prime}=F^{-1} D F^{-1 T}$. The last identity follows from

$$
D=B_{x} B_{y}^{T}-B_{y} B_{x}^{T}
$$


and the similar equation for $D^{\prime}$ and $B^{\prime}$.

Lemma 6.9. Using the previous notation, the entries of $|F| D^{\prime}$ are all polynomials in $x$ and $y$.

Proof. We introduce another matrix

$$
\tilde{B}=\left(\frac{1}{\eta^{m-1}(m-1) !} \tilde{f}_{m-1}, \ldots, \frac{1}{\eta^{0} 0 !} \tilde{f}_{0}\right)^{T}=A B,
$$

with associated matrix

$$
\tilde{D}=\left(\frac{1}{\eta^{2 m-i-j}(m-i) !(m-j) !}\left\{\tilde{f}_{m-i}, \tilde{f}_{m-j}\right\}\right) .
$$

These satisfy an equation analogous to (6-7) above, and hence $\tilde{D}=A D A^{T}$. Now,

$$
F^{-1} D F^{-1 T}=A^{-1} \tilde{F}^{-1} A D A^{T} F^{-1 T} A^{-1 T}=A^{-1} \tilde{F}^{-1} \tilde{D} \tilde{F}^{-1 T} A^{-1 T} .
$$

But the left-hand side of equation (6-9) tends to $D^{\prime}$ as $\eta \rightarrow 0$, and the right-hand side is of the form $A^{-1} D^{*} A^{-1 T}$, where $D^{*}$ has entries

$$
\frac{1}{\eta^{2 m-i-j}(m-i) !(m-j) !} \frac{\left\{\tilde{f}_{m-i}, \tilde{f}_{m-j}\right\}}{\tilde{f}_{m-i} \tilde{f}_{m-j}} .
$$

Thus, the denominator of the entries on the right-hand side of (6-9) can be at most the product of the $\tilde{f}_{i}$. Thus, in the limit, as $\eta \rightarrow 0$, the denominator of $D^{\prime}$ is at worst $f_{0}^{m}$.

Remark 6.10. In fact, the proof above shows that the denominator of $\left\{F_{i}, F_{j}\right\}$ can be no worse than $f_{0}^{k}$, where $k=\max (i, j)+1$.

In the final part of this section, we want to consider the problem of finding all polynomial vector fields $X$ which have generalized invariant algebraic curves

$$
F_{(i)}=f_{i, 0}+\varepsilon_{i} f_{i, 1}+\cdots+\varepsilon_{i}^{m_{i}-1} f_{i, m_{i}-1}
$$

of order $m_{i}$, for $i=1, \ldots, r$. We assume, as usual, that each of the $f_{i, 0}$ are irreducible.

In order not to overburden the notation, we shall drop any parentheses in the subscripts, and take the first subscript to refer to the number of the generalized curve. Otherwise, the notation is similar to the case of one curve.

Each of the $F_{i}$ defines a matrix as before, and we take the matrix $F$ to be the block-diagonal matrix with the matrices $F_{i}$ as its blocks. We take $|F|$ to be the determinant of $F$.

As in the case of one curve, each $F_{i}$ yields a family of curves $\tilde{f}_{i, j}=0$, and we get block-diagonal matrices $F^{\prime}$ and $\tilde{F}$ with blocks $F_{i}^{\prime}$ and $\tilde{F}_{i}$, respectively. 
Let $\mathscr{Y}$ be the set of pairs of integers $(i, j)$ with $1 \leq i \leq r$ and $0 \leq j \leq m_{i}-1$, which we order linearly as

$$
\left(1, m_{1}-1\right), \ldots,(1,0),\left(2, m_{2}-1\right), \ldots,(r, 0) .
$$

Using this ordering, we can define vectors $B, B^{\prime}$ and $\tilde{B}$, with entries $f_{i, j}, F_{i, j}=$ $\left[\log F_{i}\right]_{j}$ and $\tilde{f}_{i, j}$, respectively.

Furthermore, using the ordering on $\mathscr{Y}$, we can define the matrices $D, D^{\prime}$ and $\tilde{D}$ with entries $\left\{f_{s}, f_{t}\right\},\left\{F_{s}, F_{t}\right\}$ and $\left\{\tilde{f}_{s}, \tilde{f}_{t}\right\}$ respectively. (Note that these are not block-diagonal matrices.)

Finally, we take the ideal $\mathscr{I} \subset \mathbb{C}[x, y]$ to be the ideal generated by

$$
\{|F|\} \cup \bigcup_{s \in S}\left\{|F| F_{s x}\right\} \cup \bigcup_{s \in S}\left\{|F| F_{s y}\right\} \cup \bigcup_{s, t \in S}\left\{|F|\left\{F_{s}, F_{t}\right\}\right\} .
$$

The final term of this expression consists of polynomials; this is clear when $s$ and $t$ refer to different $F_{i}$, and otherwise by Lemma 6.9.

Remark 6.11. When the curves all have multiplicity one and the union of the curves $f_{i, 0}=0, i=1, \ldots, r$, form a divisor with normal crossings (each curve is smooth and all crossings are of nodal type), this ideal is just $\mathbb{C}[x, y]$. In general $\operatorname{dim}_{\mathbb{C}} \mathbb{C}[x, y] / \mathscr{I}$ represents the departure of the union of the curves from being a normal-crossing divisor.

In the case of a generalized invariant algebraic curve $f+\varepsilon g$ of order $2, \Phi$ is generated by $f^{2}, f f_{x}, f f_{y}, f_{x} g-f g_{x}, f_{y} g-f g_{y}$ and $f_{x} g_{y}-f_{y} g_{x}$. The condition that $f$ and $g$ be nonsingular and intersect transversally is sufficient to guarantee that $1 \in I$. This explains the hypotheses of Proposition 2.7.

Let $\left|F_{i}\right|=\operatorname{det} F_{i}$ and $K_{i}=|F| /\left|F_{i}\right|$. We have

$$
|F| \frac{\partial F_{i, m_{i}-1}}{\partial x}=\frac{(-1)^{m_{i}} K_{i} f_{i, 1}^{m_{i}-1}}{m_{i}-1} \frac{\partial f_{i, 0}}{\partial x} \bmod \left(f_{i, 0}\right) .
$$

If $F_{i}$ is nondegenerate or is a curve of order one, then this term is not divisible by $f_{i, 0}$ but is divisible by all the other $f_{j, 0}$ with $j \neq i$. Taking a sum of such terms we can find elements in $\mathscr{I}$ which are not divisible by any of the $f_{i, 0}$.

Finally, we take $F^{a}=|F| F^{-1}$ to be the adjugate matrix of $F$; its entries are polynomials.

Theorem 6.12. If a polynomial vector field $X$ has $r$ generalized invariant algebraic curves as above, then for any polynomial $h$ in the ideal $\Phi$ we can find a polynomial vector field $\bar{X}$ and polynomials $C_{s} \in \mathbb{C}[x, y], s \in \mathscr{Y}$, such that

$$
h X=|F| \bar{X}+\left(C_{1, m_{1}-1}, \ldots, C_{r, 0}\right) F^{a}\left(X_{1, m_{1}-1}, \ldots, X_{r, 0}\right)^{T},
$$

where $X_{s}, s \in \mathscr{Y}$, represents the vector field $X_{f_{s}}=f_{s x} \partial / \partial y-f_{s y} \partial / \partial x$. 
Proof. It clearly suffices to show that we can choose $\bar{X}$ and the $C_{s}$ for $h$ equal to each of the generators of $\mathscr{I}$. For general $h$, the result follows by the linearity of (6-10) over $\mathbb{C}[x, y]$. We suppose that $X=P \partial / \partial x+Q \partial / \partial y$.

For $h=|F|$, the result is trivial (just take $\bar{X}=X$ and all the $C_{s}$ to be zero). For the other generators, we note that the condition that the $F_{i}$ are generalized invariant algebraic curves is the same as saying

$$
X\left(F_{s}\right)=L_{s}, \quad s \in S,
$$

where the $L_{s}$ are the corresponding terms in the cofactors $L_{F_{i}}$ of $F_{i}$. The equation arises from applying $X$ to $\log F_{i}$.

Now, (6-10) is equivalent to

$$
h X=|F| \bar{X}+\left(C_{1, m_{1}-1}, \ldots, C_{n, 0}\right)\left(X_{1, m_{1}-1}^{\prime}, \ldots, X_{r, 0}^{\prime}\right)^{T},
$$

where $X_{s}^{\prime}, s \in S$, represents the vector field

$$
|F|\left\{F_{s}, \cdot\right\}=|F| F_{s x} \partial / \partial y-|F| F_{s y} \partial / \partial x .
$$

If $h=|F| F_{s x}$, we can take $\bar{X}=L_{s} \partial / \partial x, C_{s}=Q$ and $C_{s}^{\prime}=0$ for all $s^{\prime} \neq s$. From (6-11), this gives a representation of $X$ in the form (6-10).

Finally, if $h=|F|\left\{F_{s}, F_{t}\right\}$ for some $s$ and $t$, then from (6-11) we have

$$
P F_{s x}+Q F_{s y}=L_{s}, \quad P F_{t x}+Q F_{t y}=L_{t} .
$$

Solving these equations simultaneously for $P$ and $Q$, and comparing with (6-12), we find that

$$
\left\{F_{s}, F_{t}\right\} X=L_{t} X_{s}^{\prime}-L_{s} X_{t}^{\prime},
$$

which is clearly of the form (6-12).

Remark 6.13. If $L$ is the vector of cofactors $\left(L_{1, m_{1}-1}, \ldots, L_{r, 0}\right)^{T}$ so that $X(B)=$ $F L$ (or, alternatively, $X\left(B^{\prime}\right)=L$ ), then

$$
h L=F^{a} \bar{X}(B)-F D^{\prime} C,
$$

where $C=\left(C_{1, m_{1}-1}, \ldots, C_{r, 0}\right)^{T}$. In particular, we can read off the cofactors $L_{F_{i}}$ directly from this expression. The proof of (6-13) follows easily from (6-12), since

$$
h X\left(B^{\prime T}\right)=F \bar{X}\left(B^{T}\right) F^{-1 T}+\left(C_{1, m_{1}-1}, \ldots, C_{r, 0}\right) F D^{\prime},
$$

and thus

$$
h F^{-1} X(B)=h X\left(B^{\prime}\right)=F^{a} \bar{X}(B)-F D^{\prime}\left(C_{1, m_{1}-1}, \ldots, C_{r, 0}\right)^{T},
$$

which implies

$$
h X(B)=F\left(F^{a} \bar{X}(B)-F D^{\prime} C\right) .
$$


6D. Integrable multiplicity implies geometric multiplicity. Having proved Theorem 6.12 in the previous section, it is very simple to establish the following unfolding of a collection of multiple algebraic curves $F_{i}$. Let $\tilde{f}_{s}, s \in S$, be defined as in (6-3) for each curve $F_{i}$.

Theorem 6.14. Let $X$ be a vector field with generalized invariant algebraic curves $F_{i}$ of order $m_{i}$, and let $h$ be a polynomial in the ideal $\mathscr{I}$ defined in the previous section. There exists a family of polynomial vector fields $X_{\eta}$, of bounded degree and with algebraic curves $\tilde{f}_{s}, s \in S$, such that $X_{\eta}$ tends to $h X$ as $\eta$ tends to zero.

Proof. By Theorem 6.12 we have

$$
h X=|F| \bar{X}+C^{T} F^{a}\left(X_{1, m_{1}-1}, \ldots, X_{r, 0}\right)^{T},
$$

for some choice of $\bar{X}$ and $C_{s}$. Take $X_{\eta}$ to be the vector field

$$
X_{\eta}=\left|F^{\prime}\right| \bar{X}+C^{T}{F^{\prime}}^{a}\left(X_{1, m_{1}-1}, \ldots, X_{r, 0}\right)^{T} .
$$

Now, $X_{\eta}$ tends to $h X$ as $\eta$ tends to 0 . Let $A$ be the block-diagonal matrix whose blocks are the matrices $A_{i}$ associated with each curve $F_{i}$. From Lemma 6.8, $\left|F^{\prime}\right|=$ $|\tilde{F}|$, and we can write

$$
X_{\eta}=|\tilde{F}| \bar{X}+C^{T} A^{-1} \tilde{F}^{a} A\left(X_{1, m_{1}-1}, \ldots, X_{r, 0}\right)^{T} .
$$

If we take $\tilde{X}_{i, j}$ to be the vector field given by $\left(\eta^{j} j !\right)^{-1}\left\{\tilde{f}_{i, j}, \cdot\right\}$, then we have

$$
X_{\eta}=|\tilde{F}| \bar{X}+C^{T} A^{-1} \tilde{F}^{a}\left(\tilde{X}_{1, m_{1}-1}, \ldots, \tilde{X}_{r, 0}\right)^{T} .
$$

It is now clear that this vector field has the curves $\tilde{f}_{s}$ as invariants. In fact, since the matrix $\tilde{F}^{a}$ is diagonal, we can remove the factors $\left(\eta^{j} j !\right)^{-1}$ from the $\tilde{X}_{i, j}$ into the vector $C^{T} A^{-1}$, and then the system is of the form (6-10).

Corollary 6.15. If a vector field $X$ has an algebraic curve of integrable multiplicity $m$, then the geometric multiplicity of the curve is at least $m$.

Corollary 6.16. If $\mathscr{I}=(1)$, then the perturbation can be chosen to be in the class of polynomial vector fields.

We give one simple case where we can show that this is the case without calculating $\mathscr{I}$ directly.

Proposition 6.17. Suppose $f=0$ is an invariant algebraic curve of multiplicity $m$. If $f$ is nonsingular and all the critical points on $f=0$ are elementary (have at least one nonzero eigenvalue), then $\Phi=(1)$; we can therefore perturb the vector field polynomially to produce $m$ distinct invariant algebraic curves. 
Proof. It is sufficient to prove this locally, because, if the image of $\mathscr{I}$ is (1) in each local ring $\mathbb{C} \llbracket x-x_{0}, y-y_{0} \rrbracket$, then $\mathscr{I}=(1)$ by the Nullstellensatz. We can assume, without loss of generality, that the local ring is at the origin. Furthermore, a formal change of coordinates will not affect the fact that $\mathscr{I}=1$ in $\mathbb{C} \llbracket x, y \rrbracket$. Now, according to Remark 3.11, after a change of coordinates we can write $F=G F_{0}$ with $G$ a unit in $\mathbb{C} \llbracket x, y \rrbracket[\varepsilon] /\left(\varepsilon^{k}\right)$. Therefore, $|F|=|G|\left|F_{0}\right|$ and $\log F=\log G+\log F_{0}$. Thus, in the expression of $\mathscr{I}$ all the terms which involve derivatives of $G$ are multiplied by $\left|F_{0}\right|$, and so the image of the ideal $\mathscr{I}$ in $\mathbb{C} \llbracket x, y \rrbracket$ is the same as the ideal in $\mathbb{C} \llbracket x, y \rrbracket$, but calculated from $F_{0}$ instead of $F$. If we look at the forms given in Remark 3.11 and Corollary 3.12, we see that $\mathscr{I}$ is therefore equal to (1) in $\mathbb{C} \llbracket x, y \rrbracket$.

Question 6.18. Given multiple curves $f_{i}=0$, under what conditions on the $f_{i}$ and the critical points which lie on them can we guarantee that $\mathscr{I}=(1)$ ?

\section{Holonomic multiplicity}

In contrast to the previous definitions, our last definition of multiplicity seems to be genuinely weaker, and will require some extra assumptions before we can show its equivalence to the previous ones.

Consider, as in the previous section, a family of $m$ algebraic curves coalescing to a single curve which is irreducible and reduced. From an analytic standpoint, it is intuitively clear that every element of the monodromy group on this limiting curve must be of the form $x \mapsto x+O\left(x^{m}\right)$. Conversely, if the monodromy group has terms of the form $x \mapsto x+k x^{m}+\ldots$, with $k \neq 0$, then we expect that we can bifurcate $m$ leaves of the foliation from the curve by perturbation.

Unfortunately monodromy considerations alone do not seem to be strong enough to show that we can actually bifurcate this number of algebraic curves. In order to show that any bifurcating leaves are algebraic (via Chow's theorem, for example) we would need to stop the leaves from escaping at the critical points (for example, consider the pattern of leaves close to one of the separatrices of a saddle).

Thus, in what follows, we need to make some additional assumptions on the types of critical points which lie on the curve. We shall also assume that the curve is nonsingular. Fortunately, from the proof of Proposition 3.10, there are obvious assumptions we can make on the types of critical points, which also have the advantage that they can be easily checked for any given system with an algebraic curve.

We assume, as always, that $f=0$ is irreducible and reduced and that $X$ acts nontrivially on $f=0$.

7A. Some definitions. Given an algebraic curve $f=0$ in the plane and an analytic family of transversals on each point of the curve that is not in the set $S$ of singular points of the curve and the vector field, it is possible to define a map from the 
fundamental group of $\{f=0\} \backslash S$ to the group of local diffeomorphisms of $\mathbb{C}$ fixing 0 by locally lifting the paths on the curve to the leaves of the foliation via the family of transversals. Up to conjugacy, this map does not depend on the base point of the fundamental group, nor on the family of transversals chosen. For more details, see for example [Mattei and Moussu 1980]. The image of this map is called the monodromy group or holonomy group of the curve.

Definition 7.1. A curve $f=0$ is said to have holonomic multiplicity $m$, if the monodromy group of the curve lies in $A^{m} \backslash A^{m+1}$, where $A^{m}$ is the set of all germs of diffeomorphisms $\phi$ such that $\phi(x)-x=O\left(x^{m}\right)$.

As stated above, we shall assume that the curve $f=0$ is nonsingular (over the projective plane) and obeys some additional conditions on the critical points which lie on the curve. These properties are based on Corollary 3.12.

Definition 7.2. Given a nonsingular invariant algebraic curve $f=0$ of a vector field $X$, a critical point which lies on $f=0$ is said to be regular with respect to $f=0$ if it is either hyperbolic and its ratio of eigenvalues is positive, or is a saddle node and the eigenvalue associated to the direction tangent to $f=0$ is the nonzero one.

7B. Multiplicity and holonomic multiplicity. We show that the holonomic multiplicity is at least as large as the multiplicity, and that, when $f=0$ is a nonsingular curve (over the projective plane) and all the singular points on the curve, including the ones at infinity, are regular, then the two multiplicities coincide. Recall that, if the curve is multiple, the singular points on the curve are regular by Proposition 3.10 , so the assumptions we make are reasonable.

Theorem 7.3. Let $f=0$ be an invariant algebraic curve which is nonsingular as a projective curve, and suppose that all the singular points which lie on the curve are regular with respect to $f=0$ (including the points at infinity). The holonomic multiplicity of $f=0$ is the same as its (infinitesimal) multiplicity. If the curve is only irreducible and reduced, then the holonomic multiplicity is at least as great as the multiplicity, with no assumptions on the critical points.

Proof. This follows directly from the Propositions 7.4, 7.7 and 7.8 below.

Proposition 7.4. If $f=0$ is an invariant algebraic curve of multiplicity $m$, then the holonomic multiplicity of $f=0$ is at least $\mathrm{m}$.

Proof. If the multiplicity of $f=0$ is $m$, then there is a generalized invariant algebraic curve $F$ of order $m$. Let $z$ be an independent variable, define an analytic function $h(x, y, z)=F(z)$, and let $\ell(x, y, z)=L_{F}(z)$. From (3-2), we have $X(h)=$ $\ell h+O\left(z^{m}\right)$. 
We know from Proposition 3.8 that $f_{1}(p) \neq 0$ when $p \in\{f=0\} \backslash S$. Thus, given an analytic family of transversals to the curve $f=0$, we see that we can define an analytic parametrization of these transversals on a neighborhood of $\{f=0\} \backslash S$ by choosing it to be the value of $z$ such that $h(x, y, z)=0$ (existence follows from the implicit function theorem). Clearly, the curve $f=0$ corresponds to $z=0$.

Now, by differentiating $h=0$ with respect to time, we have

$$
X(h)+\frac{\partial h}{\partial z} \dot{z}=h \ell+O\left(z^{m}\right)+\left(f_{1}+O(z)\right) \dot{z}=0,
$$

which means that $\dot{z}=O\left(z^{m}\right)$ on any trajectory sufficiently close the curve $f=0$, and so the holonomy lies in $A^{m}$.

Remark 7.5. It follows that we can bound the multiplicity of a curve by looking at the monodromy of the critical points. We thus obtain an easy route to some of the results of Proposition 3.10 and Corollary 3.12. For a curve of nonzero genus, the holonomic multiplicity will contain more information than just the behavior at the critical points, as we also need to consider the holonomy about a set of curves generating the homology group.

We now set about to prove the converse, with the assumptions mentioned in the previous section. We first need a lemma to simplify the calculations a bit.

Lemma 7.6. Let $X$ be a vector field of degree $d$ (or a vector field in the form (2-2) of projective degree $d)$. The vector field obtained from $X$ by performing the projective transformation $\left(x^{\prime}, y^{\prime}\right)=\left(\ell_{1} / \ell_{3}, \ell_{2} / \ell_{3}\right)$ is of the form $\ell_{3}^{1-d} X^{\prime}$, where $X^{\prime}$ is a polynomial vector field in the form (2-2) of projective degree $d$. It is in the form (2-1) with degree $d$ if an only if $\ell_{3}=0$ is an invariant algebraic curve of the system. If $F$ is a generalized invariant algebraic curve of degree $n$ and order $k$ such that $f_{0}$ and $\ell_{3}$ are coprime, then its transform is $\ell_{3}^{-n} F^{\prime}$, where $F^{\prime}$ is also a generalized invariant algebraic curve of degree $n$ and order $k$.

Proof. The first part follows directly from calculations, and is more or less well known. For the second, let the transform of $L_{F}$ be $\ell_{3}^{1-d} L_{F}^{\prime}$ for some polynomial $L_{F}^{\prime} \in \mathbb{C}[x, y, \varepsilon]$. The equation $X(F)=F L_{F}$ thus transforms to

which simplifies to

$$
\ell_{3}^{1-d} X^{\prime}\left(\ell_{3}^{-n} F^{\prime}\right)=\ell_{3}^{-n+1-d} F^{\prime} L_{F}^{\prime},
$$

$$
\ell_{3} X^{\prime}\left(F^{\prime}\right)=n F^{\prime}\left(X^{\prime}\left(\ell_{3}\right)+L_{F}^{\prime}\right) .
$$

Hence, $\ell_{3}$ divides the term in brackets on the right-hand side, and the lemma is proved.

Proposition 7.7. If $f=0$ is a regular invariant algebraic curve of a polynomial vector field $X$ with holonomic multiplicity at least two (that is, the linear part of the holonomy group of the curve is trivial), then the multiplicity is also at least two. 
Proof. From the previous lemma, we first perform a projective transformation so that the vector field is of the form (2-2) with $h$ nonzero. We can also assume, by an appropriate choice of $\ell_{3}$, that the curve intersects the line at infinity at distinct places which are not critical points on $\mathbb{C P}(2)$. It is easy to show that the critical points at infinity for (2-2) correspond to the intersection at infinity of the curves $x b-y a=0$ and $h=0$.

Suppose that a family of transversals have been defined on $U=\{f=0\} \backslash S$, and parameterize them by $z=f(x, y)$. If $L_{f}$ is the cofactor of $f$, we can calculate the linear term of the monodromy about $f=0$ by differentiating with respect to time as before. Now, $\dot{z}=X(f)=f L_{f}=z L_{f}$, and so, given a path $\gamma$ on $f=0$, the linear term of the monodromy is given by

$$
M_{\gamma}=\exp \left(\int_{\gamma} L_{f} d t\right)
$$

If $M_{\gamma}=1$ for all curves $\gamma$ on $U$, then, fixing a point $x_{0} \in U$, the function

$$
\phi=\exp \left(\int_{p_{0}}^{p} L_{f} d t\right)
$$

is independent of the path chosen from $p_{0}$ to $p$ in $U$, and therefore gives a welldefined analytic function on $U$ (the form $d t$ being well defined and nonsingular away from the singularities on $f=0$ ). We want to show that, under the hypothesis of the proposition, $\phi$ is in fact the restriction of a polynomial $g$ to $f=0$. If so, then on $f=0$ we have $X(g)=L_{f} g$, and hence $X(g)=L_{f} g+L_{e} f$ for some polynomial $L_{e}$. This means that $\exp (g / f)$ is an exponential factor, and from Proposition 2.9 we can deduce that the degree of $g$ is in fact the same as the degree of $f$, and thus prove the result. The generalized invariant algebraic curve $f+\varepsilon g$ must be nondegenerate, since, if $g$ is a multiple of $f$, then $f$ must divide $L_{f}$, and $f+\varepsilon$ is a nondegenerate invariant algebraic curve for the system.

It only remains, therefore, to show that $\phi$ is the restriction of a polynomial to $f=0$; we can do this if we know that it is bounded as $p \rightarrow s \in S$ and has at most polynomial growth as $p \rightarrow \infty$.

From Theorem 6.12, since $f=0$ is nonsingular, there exist polynomials $A, B$ and $D$ such that

$$
X=\left(A f-D f_{y}\right) \frac{\partial}{\partial x}+\left(B f+D f_{x}\right) \frac{\partial}{\partial y},
$$

with $L_{f}=A f_{x}+B f_{y}$. The critical points which lie on $f=0$ correspond to the zeros of $D$ on $f=0$, so that $D$ does not vanish away from $S$. Thus

$$
\phi=\exp \left(\int_{p_{0}}^{p} \frac{A d y-B d x}{D}\right) .
$$


For the rest of this proof, we let $T^{i}$ represent the terms of degree $i$ in the expression $T$. If $k=\max (\operatorname{deg} A, \operatorname{deg} B, \operatorname{deg} D-1)$, then $k \geq d+1-n$. If $k>d+1-n$,

$$
A^{k} f^{n}-D^{k+1} f_{y}^{n}=0, \quad B^{k} f^{n}+D^{k+1} f_{x}^{n}=0 .
$$

Since we have assumed that the branches of $f=0$ at infinity are distinct, $f^{n}$ and $f_{x}^{n}$ are coprime, as are $f^{n}$ and $f_{y}^{n}$. Thus,

$$
A^{k}=R f_{y}^{n}, \quad D^{k+1}=R f^{n}, \quad \text { and } \quad B^{k}=-R f^{n},
$$

for some homogeneous polynomial $R$. Replacing $A, B$ and $D$ by $A-R f_{y}, B+R f_{x}$ and $D-R f$, respectively, we find that (7-2) is still satisfied, but we have reduced $\max (\operatorname{deg} A, \operatorname{deg} B, \operatorname{deg} D-1)$. Thus, by iterating this process, we can assume that

$$
k=\max (\operatorname{deg} A, \operatorname{deg} B, \operatorname{deg} D-1)=d+1-n .
$$

Now, from (2-2),

$$
A^{k} f^{n}-D^{k+1} f_{y}^{n}=x h, \quad B^{k} f^{n}+D^{k+1} f_{x}^{n}=y h,
$$

and so, eliminating $h$ and applying Euler's formula, we get

$$
x B^{k}-y A^{k}+n D^{k+1}=0 .
$$

This also gives

$$
h=\left(A^{k} f_{x}^{n}+B^{k} f_{y}^{n}\right) / n,
$$

in accordance with Proposition 2.9.

Now, the critical points at infinity are given by the intersection of $h=0$ and $x b-y a=0$. However,

$$
\begin{aligned}
x b^{d}-y a^{d}= & x\left(B^{k-1} f^{n}+B^{k} f^{n-1}+D^{k} f_{x}^{n}+D^{k+1} f_{x}^{n-1}\right) \\
& -y\left(A^{k-1} f^{n}+A^{k} f^{n-1}-D^{k} f_{y}^{n}-D^{k+1} f_{y}^{n-1}\right) \\
= & \left(x B^{k-1}-y A^{k-1}+n D^{k}\right) f^{n}+\left(x B^{k}-y A^{k}+(n-1) D^{k+1}\right) f^{n-1} .
\end{aligned}
$$

If $f^{n}=0$, then this expression vanishes if and only if $h=0$; this follows from Euler's formula and (7-4) and (7-5). Thus, the condition that there are no critical points on the branches of $f=0$ at infinity reduces to the single condition that $h$ and $f^{n}$ must be coprime.

We now want to examine the growth at infinity. Without loss of generality, we can assume that a rotation has been made so that the branch corresponds to a factor $x$ in $f^{n}$. Therefore, the condition on the critical points at infinity means that $x$ does not divide $h$, which implies, from (7-5), that it does not divide $A^{k}$. Let 
$x=a_{0}+a_{-1} y^{-1}+a_{-2} y^{-2}+\ldots$ be a parameterization of this branch as $y \rightarrow \infty$. Then, $A^{k}(x(y), y)=\alpha y^{k}+O\left(y^{k-1}\right)$ with $\alpha \neq 0$, and

$$
D^{k+1}(x(y), y)=-\left(x B^{k}-y A^{k}\right) / n=\alpha y^{k+1} / n+O\left(y^{k}\right),
$$

so that, on this branch,

$$
\frac{A d y-B d x}{D}=\left(\frac{n}{y}+O\left(y^{-2}\right)\right) d y .
$$

Hence, $\phi$ grows like $y^{n}$ as $y \rightarrow \infty$.

Finally, we consider the finite critical points on $f=0$. The problem is purely local, so we can perform a local analytic change of coordinates and, from the fact that the curve is regular, we can assume that the critical point is of the form

$$
X=(x+o(x, y)) \frac{\partial}{\partial x}+y(\lambda+O(x, y)) \frac{\partial}{\partial y} .
$$

where the curve $f=0$ corresponds to the line $y=0$. Now, let $f=y h$ with $h(0,0) \neq 0$; we have

and on $y=0$ we have

$$
L_{f}=\lambda+O(x, y)+\frac{X(h)}{h},
$$

$$
L_{f} d t=\left(\frac{\lambda}{x}+O(x)\right) d x
$$

which shows that $\phi$ must be bounded as $p$ tends to any singularity in $S$.

Proposition 7.8. If $f=0$ is a regular irreducible invariant algebraic curve of a polynomial vector field $X$ with holonomic multiplicity $m$, then the multiplicity of the curve is also $\mathrm{m}$.

Proof. We first perform a projective change of coordinates as in Proposition 7.7. The proof is by induction. Let $F$ be a nondegenerate generalized invariant algebraic curve of order $k$, and assume that the holonomy group of the curve lies in $A^{k+1}$. We want to show that $F$ can be extended to a generalized invariant algebraic curve of order $k+1$. The case $k=1$ has been proved in Proposition 7.7, so we take $k>1$.

We set $h(x, y, z)=F(z)$ and $\ell(x, y, z)=L_{F}(z)$, as in Proposition 7.4. From (3-2), we have

$$
X(h)=\ell h-z^{k}\left(f_{k-1} L_{1}+\cdots+f_{1} L_{k-1}\right)+O\left(z^{k+1}\right) .
$$

Since $k>1, F$ is a nondegenerate generalized invariant algebraic curve and, as in Proposition 7.4, we can parameterize the transversals of $U$ by the value of $z$ which makes $h=0$. Differentiating, we get $X(h)+\frac{\partial h}{\partial z} \dot{z}=h \ell-z^{k}\left(f_{k-1} L_{1}+\cdots+f_{1} L_{k-1}\right)+O\left(z^{m+1}\right)+\left(f_{1}+O(z)\right) \dot{z}=0$, 
and so, recalling that $f_{1}$ cannot vanish on $U$, we find that the holonomy is in $A^{k+1}$ if and only if

$$
\int_{\gamma} \frac{\left(f_{k-1} L_{1}+\cdots+f_{1} L_{k-1}\right)}{f_{1}} d t=0
$$

around any closed path $\gamma$ in $U$.

Thus, as in Proposition 7.7, we can fix a point $p_{0} \in U$ and define a function

$$
\phi=f_{1} \int_{p_{0}}^{p} \frac{\left(f_{k-1} L_{1}+\cdots+f_{1} L_{k-1}\right)}{f_{1}} d t
$$

which is well defined and analytic in $U$. On $f=0$ we have

$$
X(\phi)=\phi L_{0}+f_{k-1} L_{1}+\cdots+f_{1} L_{k-1} .
$$

If we can show that $\phi$ is in fact the restriction of a polynomial $f_{n}$ to $f_{0}=f=0$, then we have

$$
X\left(f_{n}\right)=f_{n} L_{0}+f_{k-1} L_{1}+\cdots+f_{1} L_{k-1}+f_{0} L_{k},
$$

for some polynomial $L_{k}$. Then we have finished, once we realize that the degree of the polynomial $f_{n}$ can be at most $n$; this follows directly from an argument similar to Proposition 2.9, considering that the highest-order terms of (7-7) involve only the terms in $f_{n}$ and $f_{0}$.

Clearly, the growth of $\phi$ at infinity can be at most polynomial, so we are left to consider the growth as $p$ tends to a point $s \in S$. Again, we can assume that the critical point is in the form (7-6) with $f=0$ corresponding to the line $y=0$. Then, on $y=0$ we can assume that $f_{1}=\alpha x^{q}+O\left(x^{q+1}\right)$ with $\alpha \neq 0$, which gives

$$
\frac{\left(f_{k-1} L_{1}+\cdots+f_{1} L_{k-1}\right)}{f_{1}} d t=\frac{O(1)}{\alpha x^{q+1}} d x .
$$

However, there can be no logarithmic terms in the integral of $\phi$, since it is single valued on $U$. Hence, when integrated, the differential gives at worst a pole of order $q$, and this is canceled by the multiplication by $f_{1}$ in the definition of $\phi$. Thus, $\phi$ is bounded as $p$ tends to $s \in S$, and has polynomial growth as $p$ tends to infinity. Therefore, it can be represented as the restriction of a polynomial function to $f=0$.

Question 7.9. To what extent can these results be generalized to singular curves, using the corresponding results of Proposition 3.13?

\section{Darboux integrability}

We finish this paper with a brief application of multiplicity to the method of Darboux integrability in the case where there are multiple curves. The next result, without taking into account the exponential factors, was proved by Darboux [1878]; 
this later improvement was made in [Christopher and Llibre 1999; 2000]. Its proof follows from straightforward computations; for more details see [Christopher and Llibre 2000].

Proposition 8.1. Let $X$ be a vector field. If $X$ admits $p$ distinct invariant algebraic curves $f_{i}=0$, for $i=1, \ldots, p$, and $q$ independent exponential factors $e_{j}$, for $j=1, \ldots, q$, then:

(a) There are $\lambda_{i}, \rho_{j} \in \mathbb{C}$ not all zero such that

$$
\sum_{i=1}^{p} \lambda_{i} L_{f_{i}}+\sum_{j=1}^{q} \rho_{j} L_{e_{j}}=0
$$

if and only if the (multivalued) function $f_{1}^{\lambda_{1}} \ldots f_{p}^{\lambda_{p}} e_{1}^{\rho_{1}} \ldots e_{q}^{\rho_{q}}$ is a first integral of the vector field $X$.

(b) There are $\lambda_{i}, \rho_{j} \in \mathbb{C}$ not all zero such that

$$
\sum_{i=1}^{p} \lambda_{i} L_{f_{i}}+\sum_{j=1}^{q} \rho_{j} L_{e_{j}}=-\operatorname{div}(X)
$$

if and only if the function $f_{1}^{\lambda_{1}} \ldots f_{p}^{\lambda_{p}} e_{1}^{\rho_{1}} \ldots e_{q}^{\rho_{q}}$ is an integrating factor of $X$.

The problem of finding first integrals or integrating factors is thus reduced to a question of linear algebra on the set of cofactors. In order to reduce the dimension of this space, we introduce the following concepts [Chavarriga et al. 1997]:

Definition 8.2. Let $X$ be a vector field of degree $d$, and $S \subset \mathbb{C}^{2}$ a finite set of points (possibly empty). The restricted cofactor space with respect to $S, \Sigma_{S}$, is defined by

$$
\Sigma_{S}=\bigcap_{p \in S} m_{p} \cap \mathbb{C}_{d-1}[x, y],
$$

where $m_{p}$ is the maximal ideal of $\mathbb{C}[x, y]$ corresponding to the point $p$.

If $S$ consists of $q$ points, then we say that they are independent with respect to $\mathbb{C}_{d-1}[x, y]$ if

$$
\sigma:=\operatorname{dim} \Sigma_{S}=\operatorname{dim} \mathbb{C}_{d-1}[x, y]-q=\frac{1}{2}(d+1)(d+2)-q .
$$

With this notation, it is easy to prove:

Theorem 8.3. Let $X$ be a vector field of degree $d$. Assume that $X$ has $r$ distinct invariant algebraic curves $f_{i}=0, i=1, \ldots, r$ (all irreducible and reduced) of multiplicity $m_{i}$, and let $N=\sum_{i} m_{i}$. Suppose, furthermore, that there are $q$ critical points $p_{1}, \ldots, p_{q}$ which are independent with respect to $\mathbb{C}_{d-1}[x, y]$, and $f_{j}\left(p_{k}\right) \neq 0$ for $j=1, \ldots, q$ and $k=1, \ldots, r$. We have:

(a) If $N \geq \sigma+2$, then $X$ has a rational first integral.

(b) If $N \geq \sigma+1$, then $X$ has a Darboux first integral. 
(c) If $N \geq \sigma$ and $\operatorname{div}(X)$ vanishes at the $p_{i}$, then $X$ has either a Darboux first integral or a Darboux integrating factor.

Proof. Each curve of multiplicity $m_{i}$ gives $m_{i}-1$ exponential factors. It is easy to see that, given that the curves $f_{i}$ are distinct and irreducible, any Darboux function constructed from them is nontrivial. The proof of (b) and (c) follows from counting dimensions and applying Proposition 8.1. One has just to observe that, by Proposition 3.9, all possible cofactors are contained in $\Sigma_{S}$.

When $N$ is at least $\sigma+2$, from (b) we can obtain two independent Darboux first integrals, say $H_{1}$ and $H_{2}$. We can see easily that the integrating factor $R_{i}$ associated to $\log H_{i}$ is a rational function. Since the quotient of two integrating factors is a first integral, the statement (a) follows from the independence of $H_{1}$ and $H_{2}$.

Our final theorem is probably the most useful one for real applications of Darboux integrability to specific families of vector fields.

Theorem 8.4. Suppose we have an algebraic family of vector fields $X_{t}$, with $t$ lying in a neighborhood of $t=0$, such that the vector fields have a collection of invariant algebraic curves of constant degree varying analytically with $t$ and which give a Darboux integrating factor (respectively, first integral) as in Proposition 8.1 for $t \neq 0$. Suppose, furthermore, that at $t=0$ all of these curves remain irreducible and reduced, though they are allowed to coalesce. Then $X_{0}$ also possesses a Darboux integrating factor (respectively, first integral).

Proof. Since the problem is analytic, we can restrict attention to a sufficiently small neighborhood of $t=0$ so that, for all $t \neq 0$, we have a fixed number of invariant algebraic curves, say $N$, and the degree of $X_{t}$ is bounded by some constant, say $d$. By Proposition 6.5, we still have $N$ curves and exponential factors when $t=0$.

Let $\Sigma_{t} \subset \mathbb{C}_{d-1}[x, y]$ be the space of cofactors (including the cofactors of the exponential factors when $t=0$ ) of these curves, and denote its dimension by $N_{t}$. Restricting $t$ to a smaller neighborhood of 0 if necessary, we can assume that $N_{t}$ is a constant for $t \neq 0$.

The $\Sigma_{t}$ are just the sum of the subspaces $\sigma_{t}$ given in Remark 6.6 for each distinct curve at $t=0$. From that remark we know that the limit of the spaces $\Sigma_{t}$ (in the space of $N$-dimensional subspaces of $\mathbb{C}_{d-1}[x, y]$ ) must contain $\Sigma_{0}$. Thus, $N_{0} \leq N_{t}$ for $t \neq 0$.

We consider only those curves which appear in the Darboux integrating factor or first integral. In the case where there is a Darboux first integral for $t \neq 0$, the number of curves $N$ is greater than $N_{t}$ for $t \neq 0$, and hence, in the limit, the number of curves and exponential factors (also $N$ ) must be greater than the dimension of the space of their cofactors $N_{0}$. This gives us a Darboux first integral at $t=0$.

In the case of a Darboux integrating factor for $t \neq 0$, we define $D_{t}$ to be the one-dimensional subspace of $\mathbb{C}_{d-1}[x, y]$ generated by the divergence of the vector 
field $X_{t}$. We also define $\tilde{\Sigma}_{t}$ to be the sum of $\Sigma_{t}$ and $D_{t}$. Its limit must contain the sum of the space $D_{0}$ and the space of cofactors of the invariant algebraic curves and their exponential factors. Arguing as above, the number of invariant algebraic curves for $t \neq 0$ must be greater than the dimension of $\tilde{\Sigma}_{t}$ for $t \neq 0$, and hence, when $t=0$, the number of curves and exponential factors must be greater than the dimension of the space generated by their cofactors and the divergence of the vector field. This gives us either a Darboux integrating factor or first integral for $t=0$ (and, in the latter case, trivially an integrating factor).

Question 8.5. How do these results extend to the case where the invariant curves may be reducible or nonreduced?

\section{Final remarks}

Although we studied vector fields in the complex plane, we suspect that many of the results remain valid in the case of real vector fields [Man and MacCallum 1997], or codimension-1 foliations on $\mathbb{C P}(n)$ [Jouanolou 1979].

Our main desire, however, is to see how these results extend to multiple curves based on reducible or nonreduced curves. We hope to return to this problem in a later paper.

\section{Acknowledgments}

We want to express our gratitude to Dana Schlomiuk and Nicolas Vulpe for providing examples that permitted us to test the different notions of multiplicity.

Llibre and Pereira thank the organizers of the Workshop on asymptotic series, differential algebra and finiteness problems in nonlinear dynamical systems held at the Centre de Recherches Mathématiques, Université de Montréal, where the initial ideas for the paper were conceived.

\section{References}

[Chavarriga et al. 1997] J. Chavarriga, J. Llibre, and J. Sotomayor, "Algebraic solutions for polynomial systems with emphasis in the quadratic case", Exposition. Math. 15:2 (1997), 161-173. MR 98h:34053 Zbl 0895.34029

[Christopher 1994] C. J. Christopher, "Invariant algebraic curves and conditions for a centre", Proc. Roy. Soc. Edinburgh Sect. A 124:6 (1994), 1209-1229. MR 95m:34052 Zbl 0821.34023

[Christopher 1999] C. Christopher, "Liouvillian first integrals of second order polynomial differential equations", Elec. J. Differential Equations (1999), \#49, 1-7. MR 2000i:34017 Zbl 0939.34002

[Christopher and Llibre 1999] C. Christopher and J. Llibre, "Algebraic aspects of integrability for polynomial systems”, Qual. Theory Dyn. Syst. 1:1 (1999), 71-95. MR 2001b:34060

[Christopher and Llibre 2000] C. Christopher and J. Llibre, "Integrability via invariant algebraic curves for planar polynomial differential systems", Ann. Differential Equations 16:1 (2000), 5-19. MR 2001g:34001 Zbl 0974.34005 
[Christopher et al. 2002] C. Christopher, J. Llibre, C. Pantazi, and X. Zhang, "Darboux integrability and invariant algebraic curves for planar polynomial systems", J. Phys. A 35:10 (2002), 2457-2476. MR 2003c:34037 Zbl 1012.34035

[Darboux 1878] G. Darboux, "Mémoire sur les équations différentielles algébriques du premier ordre et du premier degré.", Bull. Sci. Math. (2) 2 (1878), 60-96, 123-144, 151-200. JFM 10.0214.04

[Dobrovol'skii et al. 1998] V. A. Dobrovol'skii, N. V. Lokot', and J.-M. Strelcyn, "Mikhail Nikolaevich Lagutinskii (1871-1915): un mathématicien méconnu", Historia Math. 25:3 (1998), 245-264. MR 2000g:01038 Zbl 0914.01023

[van den Essen 1979] A. van den Essen, "Reduction of singularities of the differential equation $A d y=B d x "$, pp. 44-59 in Équations différentielles et systèmes de Pfaff dans le champ complexe (Sem., Inst. Rech. Math. Avancée, Strasbourg, 1975), edited by R. Gérard and J.-P. Ramis, Lecture Notes in Math. 712, Springer, Berlin, 1979. MR 82m:34007 Zbl 0418.34008

[Gröbner and Knapp 1967] W. Gröbner and H. Knapp, Contributions to the method of Lie series, B. I. Hochschulskripten 802/802a, Bibliographisches Institut, Mannheim, 1967. MR 39 \#6027 Zbl 0168.33001

[Jouanolou 1979] J. P. Jouanolou, Équations de Pfaff algébriques, Lecture Notes in Mathematics 708, Springer, Berlin, 1979. MR 81k:14008 Zbl 0477.58002

[Kleiman 1998] S. L. Kleiman, "Bertini and his two fundamental theorems", Rend. Circ. Mat. Palermo (2) Suppl. 55 (1998), 9-37. MR 99m:14001 Zbl 0926.14001

[Lins Neto and Scárdua 1997] A. Lins Neto and B. Scárdua, "Folheações algébricas complexas", Lecture notes, $21^{\circ}$ Colóquio Brasileiro de Matemática, IMPA, Rio de Janeiro, 1997.

[Man and MacCallum 1997] Y.-K. Man and M. A. H. MacCallum, "A rational approach to the Prelle-Singer algorithm”, J. Symbolic Comput. 24 (1997), 31-43. MR 98g:12009 Zbl 0922.12007

[Mattei and Moussu 1980] J.-F. Mattei and R. Moussu, "Holonomie et intégrales premières", Ann. Sci. École Norm. Sup. (4) 13:4 (1980), 469-523. MR 83b:58005 Zbl 0458.32005

[Pereira 2001] J. V. Pereira, "Vector fields, invariant varieties and linear systems", Ann. Inst. Fourier (Grenoble) 51:5 (2001), 1385-1405. MR 2003e:32041 Zbl 01654606

[Poincaré 1891] H. Poincaré, "Sur l'integration algébrique des équations différentielles du premier ordre et du premier degré”, Rend. Circ. Mat. Palermo 5 (1891), 161-191. JFM 23.0319.01

[Poincaré 1897] H. Poincaré, "Sur l'integration algébrique des équations différentielles du premier ordre et du premier degré", Rend. Circ. Mat. Palermo 11 (1897), 193-239. JFM 28.0292.01

[Prelle and Singer 1983] M. J. Prelle and M. F. Singer, "Elementary first integrals of differential equations", Trans. Amer. Math. Soc. 279:1 (1983), 215-229. MR 85d:12008 Zbl 0527.12016

[Schlomiuk 1997] D. Schlomiuk, "Basic algebro-geometric concepts in the study of planar polynomial vector fields", Publ. Mat. 41:1 (1997), 269-295. MR 99c:58120 Zbl 0889.34027

[Schlomiuk and Vulpe 2004] D. Schlomiuk and N. Vulpe, "Planar quadratic vector fields with invariant lines of total multiplicity at least five", Qual. Theory Dyn. Syst. 5:1 (2004), 135-194. MR 2197428 Zbl 05024706

[Singer 1992] M. F. Singer, "Liouvillian first integrals of differential equations", Trans. Amer. Math. Soc. 333:2 (1992), 673-688. MR 92m:12014 Zbl 0756.12006

[Walcher 2000] S. Walcher, "Plane polynomial vector fields with prescribed invariant curves", Proc. Roy. Soc. Edinburgh Sect. A 130:3 (2000), 633-649. MR 2001h:32037 Zbl 0961.34017

[Żołądek 1992] H. Żołąek, “The solution of the center-focus problem”, preprint, University of Warsaw, 1992. 
Received May 5, 2005.

COLIN CHRISTOPHER

DEPARTMENT OF MATHEMATICS AND STATISTICS

UNIVERSITY OF PLYMOUTH

PLYMOUTH PL2 3AJ

UNITED KINGDOM

c.christopher@plymouth.ac.uk

JAUME LLIBRE

DEPARTAMENT DE MATEMÀtiques

UNIVERSITAT AUTÒNOMA DE BARCELONA

08193 - BELLATERRA BARCELONA

SPAIN

jllibre@mat.uab.es

JoRge VitóRIO PEREIRA

Instituto de Matemática Pura E APliCAdA

ESTRADA DONA CASTORINA, 110

JARDIM BOTÂNICO

22460-320 RIO DE JANEIRO, RJ

BRASIL

jvp@impa.br 